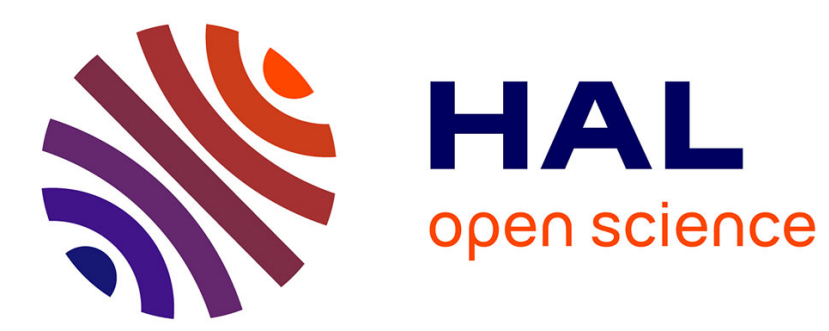

\title{
Computational Drag Prediction of the DPW4 configuration using the Far-Field Approach
}

\author{
David Hue, Sébastien Esquieu
}

\section{To cite this version:}

David Hue, Sébastien Esquieu. Computational Drag Prediction of the DPW4 configuration using the Far-Field Approach. AIAA Journal, 2011, 48 (5), pp.1658-1670. hal-01894113

\section{HAL Id: hal-01894113 \\ https://hal.science/hal-01894113}

Submitted on 23 Nov 2018

HAL is a multi-disciplinary open access archive for the deposit and dissemination of scientific research documents, whether they are published or not. The documents may come from teaching and research institutions in France or abroad, or from public or private research centers.
L'archive ouverte pluridisciplinaire HAL, est destinée au dépôt et à la diffusion de documents scientifiques de niveau recherche, publiés ou non, émanant des établissements d'enseignement et de recherche français ou étrangers, des laboratoires publics ou privés. 


\title{
Computational Drag Prediction of the DPW4 configuration using the Far-Field Approach
}

\author{
David HUE ${ }^{1}$ and Sébastien ESQUIEU ${ }^{2}$ \\ ONERA, Meudon, 92190, France
}

This paper presents the computational studies done at ONERA in the context of the $4^{\text {th }}$ AIAA CFD Drag Prediction Workshop. Furthermore, it gives a detailed description of the far-field methods developed in the Applied Aerodynamics Department. Concerning the DPW4 configuration, a grid convergence study and a downwash study are proposed. Then, the effects due to Mach and Reynolds numbers variations are quantified. All the multiblock structured grids used in this work have been provided by Boeing to the DPW community. All the RANS computations are performed by using the ONERA-elsA solver with the Spalart-Allmaras turbulence model, the solutions are post-processed with the ONERA-ffd72 far-field drag extraction tool. Concerning drag predictions, a very good agreement has been observed between ONERA-elsA results and the near-field drag coefficients (pressure and friction) computed by other DPW4 participants such as Boeing or Airbus. Moreover, the far-field software ffd72 gives to ONERA the singular capability to determine the values of the different physical drag components (viscous, wave and lift-induced productions). Concerning the pitching moment, ONERA results are very close to Boeing, Airbus or DLR predictions.

$$
\begin{array}{lll}
M a & =\text { Mach number } & \text { Nomenclature } \\
\operatorname{Re} & =\text { Reynolds number } \\
\alpha & =\text { angle of attack } \\
\mathrm{c} & =\text { wing chord }
\end{array}
$$

\footnotetext{
1 Engineer, Civil Aircraft Unit, Applied Aerodynamics Department, ONERA, 8 rue des Vertugadins 92190 MEUDON (FRANCE), david.hue@ onera.fr, AIAA Member

2 Engineer, Civil Aircraft Unit, Applied Aerodynamics Department, ONERA, 8 rue des Vertugadins 92190 MEUDON (FRANCE)
} 


$$
\begin{aligned}
& \text { Sref }=\text { reference surface } \\
& b \quad=\text { wing span } \\
& \mathrm{Cp} \quad=\text { pressure coefficient } \\
& C f=\text { skin friction coefficient } \\
& C L=\text { lift coefficient } \\
& C D p \quad=\text { pressure drag coefficient } \\
& C D f=\text { friction drag coefficient } \\
& \text { CDnf = near-field drag coefficient } \\
& C D v=\text { viscous drag coefficient } \\
& C D v p=\text { viscous pressure drag coefficient } \\
& C D w \quad=\quad \text { wave drag coefficient } \\
& C D i=\text { induced drag coefficient } \\
& \text { CDff = far-field drag coefficient } \\
& C D s p=\text { spurious drag coefficient } \\
& C M \quad=\text { pitching moment } \\
& \text { RANS = Reynolds Averaged Navier-Stokes } \\
& S A=\text { Spalart-Allmaras turbulence model } \\
& W B \quad=\text { wing } / \text { body } \\
& W B H=\text { wing } / \text { body } / \text { horizontal tail } \\
& i H=\text { horizontal tail deflection } \\
& \text { HTP }=\text { horizontal tail plane }
\end{aligned}
$$

\section{Introduction}

\footnotetext{
B

ASED on the successful development of numerical methods for solving the Reynolds-Averaged Navier-Stokes equations and the availability of computational resources, it is today possible to compute the flow for complete aircraft. However, the accuracy of the computed data such as aircraft drag and moment that is needed for improved design still depends on turbulence models, transition, or grid size and topology and is sometimes not sufficient for all points of the flight envelope.
} 
In this context, the international Drag prediction Workshop series were initiated by a working group of AIAA Applied Aerodynamics Technical Committee members focusing on CFD drag prediction. In 2009, a new benchmark experiment on a publicly available geometry suitable for cooperative assessment and validation of aerodynamic tools was proposed. A new so-called NASA Common Research Model configuration (CRM) has been designed based on the lessons learnt from the former DPW sessions.

In order to enhance its knowledge concerning the capabilities of RANS software to evaluate the drag and pitching moment of transport aircraft, the Civil Aircraft Unit of the Applied Aerodynamics Department and the Computational Fluid Dynamics and Aeroacoustics Department of ONERA participated in the $4^{\text {th }}$ AIAA Drag Prediction Workshop. The focus of this DPW4 was on drag and moment prediction accuracy for the new wing/body/horizontal-tail CRM configuration close to the design Mach number.

Preliminary results were shown by ONERA ${ }^{1}$ during the DPW4 conference held in San Antonio, Texas, in June 2009. This paper describes these results with more details or far-field analyses and it also presents the additional work that has been performed since then. First, it proposes a quite complete description of the ONERA far-field method. Then, a grid convergence study (multiblock structured grids from $5.10^{6}$ nodes to $50.10^{6}$ nodes) and a downwash study (polars with tail-off and tail-on at 3 deflection angles) are carried out. Finally, the Mach and Reynolds numbers effects are investigated.

All the multiblock structured grids used in this work were provided by Boeing to the DPW4 community. All the RANS computations are performed with the ONERA-elsA solver and the Spalart-Allmaras turbulence model. The solutions are post-processed with the ONERA-ffd72 far-field tool which permits to quantify and to locate the different physical drag productions, thus giving data of prime importance to improve the aircraft design process.

\section{NASA CRM configuration, grids, aerodynamic solver and far-field approach}

\section{A. NASA CRM configuration}

The focus of the fourth Drag Prediction Workshop is on drag and moment prediction accuracy on the wingbody-horizontal tail NASA Common Research Model configuration. This new relevant open geometry was designed by a NASA Technical Working Group considering the lessons learned during the former DPW series. Within this background, "The Boeing company took the lead on detailed aerodynamic design of the CRM, while NASA 
FA/SFW (Fundamental Aeronautics / Subsonic Fixed Wing) project took the lead on model design, fabrication and testing of the CRM"2.

As a result, the NASA-CRM configuration has the following characteristics:

- Conventional low-wing configuration;

- A nacelle/pylon installation can be included;

- $\quad$ A design Mach number of 0.85 was established;

- The horizontal tail is suitably sized for typical stability and control requirements;

- The fuselage is representative of a wide/body commercial aircraft;

- $\quad \mathrm{CAD}$ references and pitching-axis are shown in Figure 1.
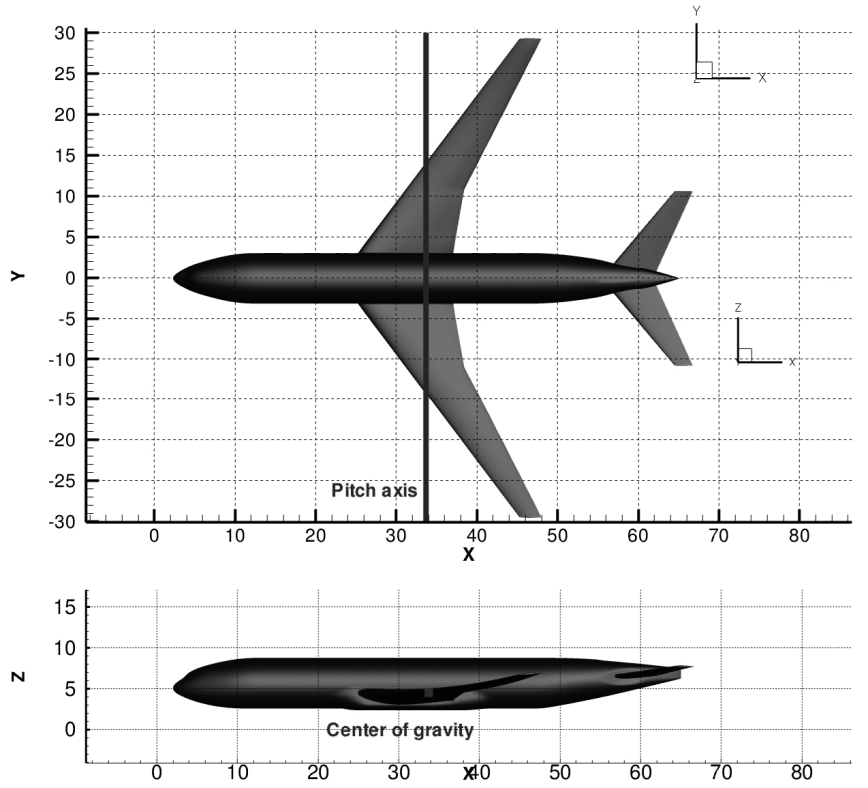

Figure 1. CRM wing / body / horizontal tail plane configuration $\left(\mathrm{iH}=0.0^{\circ}\right)$.

In this study concerning DPW4, only the clean wing without nacelle/pylon group is considered and all the study will be devoted to the wing/body/tail-off and wing/body/horizontal-tail configurations. 
The reference geometry is defined by:

- Mean-aerodynamic chord c $=7.00532 \mathrm{~m}$;

- $\quad$ Reference surface Sref $=383.68956 \mathrm{~m}^{2}$ (full-model);

- $\quad$ Semi-span $b / 2=29.38145 \mathrm{~m}$;

- $\quad$ Aspect Ratio AR = 9.0;

- Moments Xref $=33.67786 \mathrm{~m}$, Yref $=0.0 \mathrm{~m}$, Zref $=4.51993 \mathrm{~m}$.

The aerodynamic conditions for this CRM-NASA model are the following:

- Mach number: $\mathrm{Ma}=0.85$ corresponds to the design point but computations and will tunnel tests will investigate range of Mach number from 0.70 to 0.92 ;

- Reynolds number $\mathrm{Re} / \mathrm{c}=5.10^{6}$ corresponding to NASA Ames 11-ft transonic wind tunnel conditions and Reynolds number from 3 to $30.10^{6}$ in the NASA National Transonic Facility (NTF) at Langley.

\section{B. Structured grids}

To allow participation as large as possible, different participants generated grids and made them available for all the partners. In this work, the Boeing multi-block structured grids generated with Zeus have been used to perform the CFD computations by converting the available Plot3D files into CGNS files. Boeing made available 7 multiblock structured grids as indicated in the following Table 1:

Table 1. CRM configuration - Multi-block structured grids provided by Boeing.

\begin{tabular}{|c|c|c|}
\hline Coarse grid & $\mathrm{CRM} \mathrm{W} / \mathrm{B} / \mathrm{H}\left(\mathrm{iH}=0.0^{\circ}\right)$ & 4.9 million nodes \\
\hline Medium grid & $\mathrm{CRM} W / \mathrm{B}$ tail off & 11.1 million nodes \\
\hline Medium grid & $\mathrm{CRM} \mathrm{W} / \mathrm{B} / \mathrm{H}\left(\mathrm{iH}=0.0^{\circ}\right)$ & 11.2 million nodes \\
\hline Medium grid & $\mathrm{CRM} \mathrm{W} / \mathrm{B} / \mathrm{H}\left(\mathrm{iH}=-2.0^{\circ}\right)$ & 11.2 million nodes \\
\hline Medium grid & $\mathrm{CRM} \mathrm{W} / \mathrm{B} / \mathrm{H}\left(\mathrm{iH}=+2.0^{\circ}\right)$ & 11.2 million nodes \\
\hline Medium-fine grid & $\mathrm{CRM} \mathrm{W/B} / \mathrm{H}\left(\mathrm{iH}=0.0^{\circ}\right)$ & 26.0 million nodes \\
\hline Fine grid & $\mathrm{CRM} \mathrm{W} / \mathrm{B} / \mathrm{H}\left(\mathrm{iH}=0.0^{\circ}\right)$ & 47.8 million nodes \\
\hline
\end{tabular}

These meshes are made of 4 H-type structured blocks, point matched multiple block grids for CFL3D or TLNS3D type of codes. Through this strategy, a precise control on grid quality, such as grid spacing, stretching ratio and grid orthogonality near configuration surfaces is achieved (see Figure 2). 


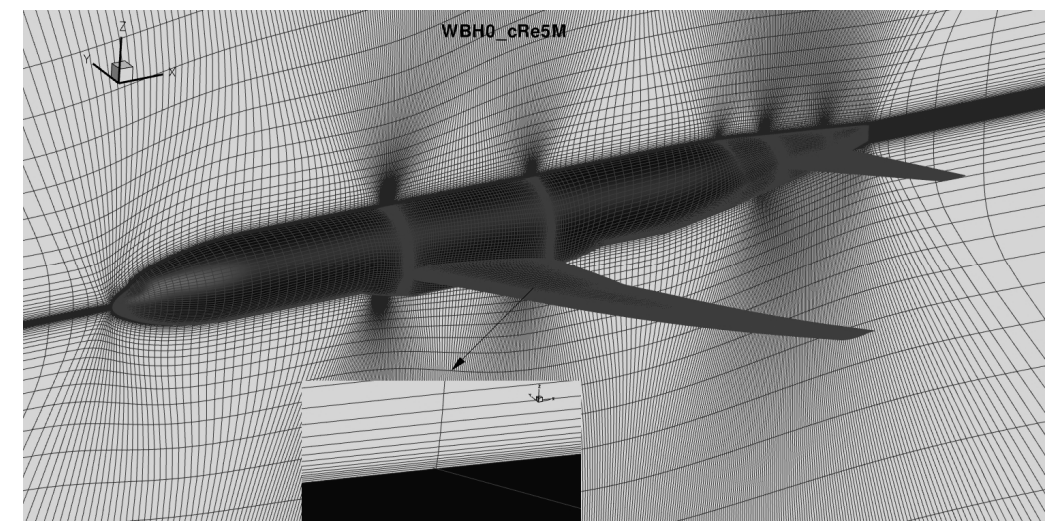

Figure 2. CRM wing / body / horizontal tail plane configuration ( $\mathrm{iH}=0.0^{\circ}$ ) - Multi-block structured grids Skin mesh and boundary layer in the inner wing region.

\section{CFD solver: ONERA-elsA}

Structured RANS computations are performed with the ONERA-elsA code ${ }^{3,4}$. This software uses a cell-centered finite-volume discretisation on structured multi-block meshes. Time integration is carried out by a backward-euler scheme with implicit LU-SSOR relaxation. Spatial discretisation is realized through a central Jameson scheme with artificial viscosity. Multigrid techniques are used to accelerate the convergence. All the computations are performed in fully turbulent conditions. Turbulence effects are simulated by the one-equation Spalart-Allmaras model. The structured elsA computations are performed on a NEC SX-8 and a Bull Novascale platforms in sequential and parallel modes.

\section{Far-field extraction method}

Here are presented the far-field drag extraction approach and the different methods which have been developed in the Applied Aerodynamics Department of ONERA in order to make use of it. Most of the following sub-sections are inspired or extracted from the reference ${ }^{5}$ by D. Destarac, with his authorization.

\section{Near-field drag}

If $\mathrm{S}_{\mathrm{A}}$ is the surface of a closed body with unit normal vector $\vec{n}$ oriented from inside the fluid towards the solid body, the $x$-direction (vector $\vec{i}$ ) being that of the velocity at upstream infinity (aerodynamic referential, not aircraft referential), total drag (D) computed following the near-field approach ( $\left.\mathrm{Dnf}_{\mathrm{nf}}\right)$, the sum of pressure drag $\left(\mathrm{D}_{\mathrm{p}}\right)$ and of friction drag (Df), is given by the formula: 


$$
D=D_{n f}=D_{p}+D_{f}=\iint_{S_{A}}\left[\left(p-p_{\infty}\right) n_{x}-\left(\vec{\tau}_{x} \cdot \vec{n}\right)\right] d S
$$

No further development is necessary. This mechanical decomposition is straightforward and needs no assumptions concerning the flow.

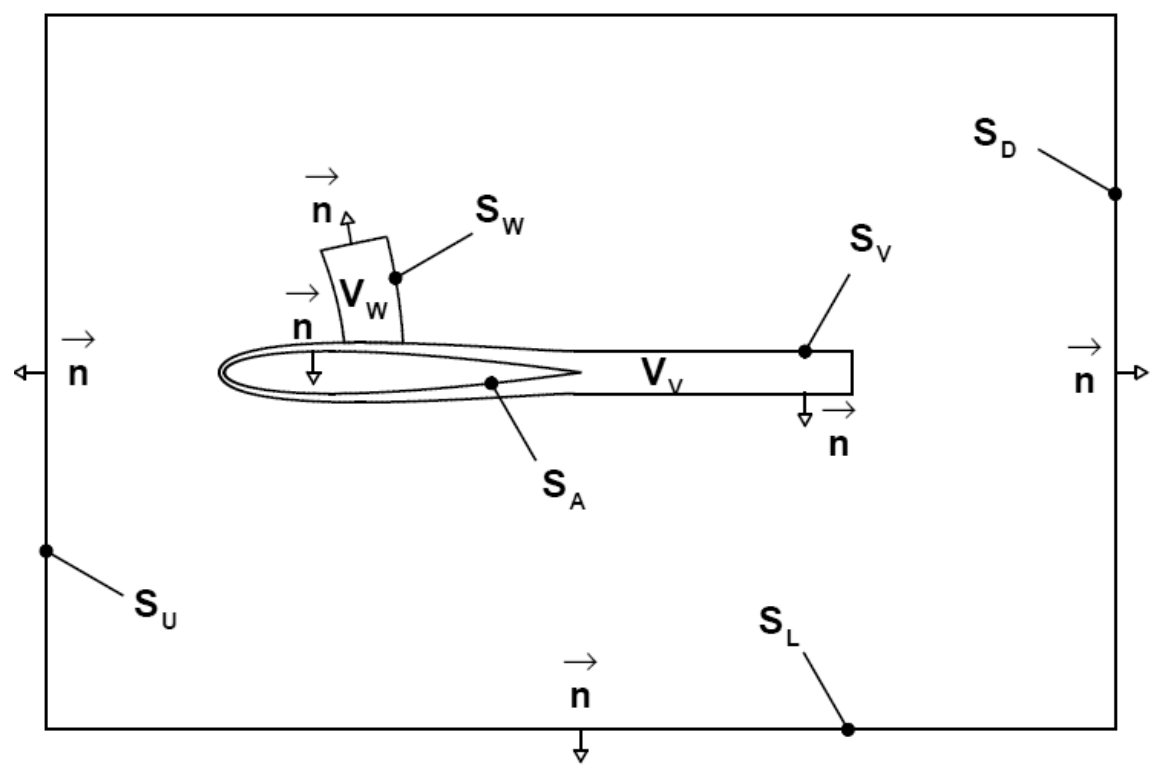

Figure 3 : Control surfaces and volumes for far-field drag integration

\section{Far-field drag}

Drag given by an expression involving surface or volume integrals within the flowfield and not only at the fluid / solid interface, is called far-field drag (nevertheless it may involve some integrals over this interface as part of the formulation).

It will be shown in this theoretical section, that the far-field drag theory involves many assumptions and that the physical far-field drag components are introduced through definitions affected by some form of arbitrariness. The theory presented here follows the innovative one of Van der Vooren ${ }^{6}$ with some minor deviations. It is applicable to steady flows described by the RANS equations.

\section{Application of conservation laws}

A simple expression for far-field total drag can be obtained by introducing the following vector 


$$
\vec{f}=-\rho\left(u-u_{\infty}\right) \vec{q}-\left(p-p_{\infty}\right) \vec{i}+\vec{\tau}_{x}(2)
$$

Combination of mass conservation and momentum theorem in the freestream velocity direction $\mathrm{x}$ gives to $\sim \mathrm{f}$ the following property

$$
\operatorname{div}(\vec{f})=0(\mathbf{3})
$$

If $\mathrm{S}_{\mathrm{A}}$ is the surface of the aircraft and So an arbitrary closed surface within the flow-field, enveloping the aircraft (no intersection between $\mathrm{S}_{\mathrm{A}}$ and So), the divergence theorem and eq.(3) allow to write

$$
\iint_{S_{A} \cup S_{0}}(\vec{f} \cdot \vec{n}) d S=0
$$

with unit normal vector $\vec{n}$ oriented from inside the fluid towards the body on $\mathrm{S}_{\mathrm{A}}$ and from inside the fluid control volume bound by So outwards on this surface.

From the near-field definition, eq.(1), the definition of vector $\vec{f}$, eq.(2) and the property $(\vec{q} \cdot \vec{n})=0$ on the aircraft surface $\mathrm{S}_{\mathrm{A}}$, there can be written

$$
\iint_{S_{A}}(\vec{f} \cdot \vec{n}) d S=-D(\mathbf{5})
$$

such that the following far-field expression of total drag can be derived from eq.(4)

$$
D_{n f}=D_{p}+D_{f}=\iint_{S_{0}}(\vec{f} \cdot \vec{n}) d S=D_{f f}(\mathbf{6})
$$

This expression is exact. It ensures an exact near-field / far-field drag balance, Dnf $=$ Dff, but gives even less information than the near-field expression, eq.(1). It is only the starting point for the physical drag breakdown theory.

\section{Far-field drag in the absence of trailing vorticity}

The particular case of a flow without trailing vorticity (for example but not necessarily two-dimensional) is considered in this subsection. 
In eq.(6), take So with such sufficient extension into the far-field, that the flow is in the freestream state on the upstream and lateral parts of $\mathrm{So}_{\mathrm{S}} \mathrm{S}_{\mathrm{U}} \cup \mathrm{SL}_{\mathrm{L}}$ (figure 2), $\mathrm{u}=\mathrm{u}_{\infty}, \mathrm{v}=\mathrm{w}=0, \mathrm{p}=\mathrm{p}_{\infty}$; and that $[\tau]=0$ on the whole surface. This theory requires the assumption that viscous phenomena are confined within volumes of finite extent, surrounded by an outer volume of truly inviscid flow ${ }^{6}$. The assumption that the shock waves do not extend to infinity (subsonic freestream) is also necessary to ensure a non perturbed state on SL.

In the absence of trailing vorticity, $\mathrm{v}=\mathrm{w}=0$ on the downstream part $\mathrm{SD}_{\mathrm{D}}$ of the surface $\left(\mathrm{SO}_{\mathrm{O}}=\mathrm{SU}_{U} \cup \mathrm{SL}_{\mathrm{L}} \cup \mathrm{SD}_{\mathrm{D}}\right.$ ). Then, the momentum theorem projected on the $y$ and $z$ directions implies (with $[\tau]=0$ ): $p=p_{\infty}$ on $S_{D}$. With the following definitions introduced by Van der Vooren ${ }^{7}$,

$$
\begin{aligned}
& \bar{u}=u\left[p=p_{\infty}, v=0, w=0, \Delta H, \Delta s\right], \Delta \bar{u}=\bar{u}-u_{\infty} \text { (7) } \\
& \Delta \bar{u}=u_{\infty} \sqrt{1+2 \frac{\Delta H}{u_{\infty}^{2}}-\frac{2}{(\gamma-1) M_{\infty}^{2}}\left[\left(e^{\frac{\Delta s}{r}}\right)^{\frac{\gamma-1}{\gamma}}-1\right]-u_{\infty}(\mathbf{8})}
\end{aligned}
$$

where $\Delta H$ and $\Delta s$ are respectively the variation of stagnation enthalpy and the variation of entropy relative to the freestream state.

The issue of the existence of this quantity, i.e. of the sign of the quantity under the square root symbol, has been discussed by Méheut ${ }^{8}$. This quantity will be positive only if the local stagnation pressure is higher than the freestream static pressure. In a solution to the RANS equations, the Mach number is equal to zero on the surface of the body. Hence, local stagnation pressure will be equal to local static pressure. Since somewhere on the body the local static pressure will be lower than the freestream static pressure (for a lifting airfoil over the larger part of the upper surface), there will certainly be areas in the flow where $\Delta \bar{u}$ does not exist. However, these areas are located close to the body, and there should be no difficulty in finding a surface So where $\Delta \bar{u}$ is defined everywhere.

On So, with $\mathrm{p}=\mathrm{p}_{\infty}, \vec{\tau}_{x}=\overrightarrow{0}$ and eq.(8), the expression for $\vec{f}$, eq.(2), will reduce to $-\rho \Delta \vec{u} \vec{q}$. It is convenient, following Van der Vooren, to introduce the notation

$$
\vec{f}_{v w}=-\rho \Delta \bar{u} \vec{q} \text { (9) }
$$

Then, eq.(6) takes the form 


$$
D=D_{f f}=\iint_{S_{0}}\left(\vec{f}_{v w} \cdot \vec{n}\right) d S(\mathbf{1 0})
$$

For reasons (formal, not physical), which will be given later, the term $\vec{\tau}_{x}$ in the expression of $\vec{f}$, which is negligible on So, may also be included in the far-field drag integral by putting :

$$
\vec{f}_{v w}^{*}=-\rho \Delta \bar{u} \vec{q}+\vec{\tau}_{x}(\mathbf{1 1})
$$

thus replacing eq.(10) by

$$
D=D_{f f}=\iint_{S_{0}}\left(\vec{f}_{v w}^{*} \cdot \vec{n}\right) d S(\mathbf{1 2})
$$

In a flow without trailing vorticity, total drag is the sum of viscous drag $D_{v}$ and wave drag $D_{w}$, the two drag components produced through irreversible thermodynamic processes. With the assumption that viscous phenomena and shock waves are confined to volumes of finite extent $\mathrm{Vv}, \mathrm{Vw}$ included in the volume Vo bound by So, the flow outside $\mathrm{Vv} \cup \mathrm{Vw}$ may be considered as inviscid and not subject to heat conduction. Then, $\Delta s$ and $\Delta H$ will not be allowed to vary along streamlines, and in this outer volume, there will be no production of $\Delta \bar{u}$. Also, outside $\mathrm{Vv}$ $\cup \mathrm{Vw}, \vec{\tau}_{x}$ will be equal to zero. Then, if Svw is the outer boundary of $\mathrm{Vv} \cup \mathrm{Vw}$, the integration in eqs.(10), (12) may be restricted to Svw,

$$
D=D_{f f}=D_{v}+D_{w}=\iint_{S_{V W}}\left(\vec{f}_{v w}^{*} \cdot \vec{n}\right) d S(\mathbf{1 3})
$$

and a similar formula for the expression with $\vec{f}_{v w}$.

\section{Definition of viscous drag and wave drag}

From this point, some differences, more formal than theoretical, will appear between the present formulation and the original article of Van der Vooren ${ }^{6}$. The formulation with $\vec{f}_{v w}^{*}$ will be used instead of the formulation with $\vec{f}_{v w}$. Surface integrals will be used instead of volume integrals obtained using the 
divergence theorem for two reasons. First, if vector $\vec{f}$ has indeed a divergence in the whole field, it is theoretically not sure that at discontinuities it should also be the case for vector $\vec{f}_{v w}^{*}$. In the numerical practice, the objection is anyway irrelevant, a true numerical discontinuity being the case in which variables at a given control point has two different values, which does not happen in usual computational fluid dynamics. Second, since there are areas in the flowfield where $\Delta \bar{u}$, and consequently $\vec{f}_{v w}^{*}$, do not exist, it is more straightforward to use surface integrals, with the assumption that the integration surfaces do not intersect such areas (which can be forced in the implementation of drag extraction), than to introduce a prolongation of $\Delta \bar{u}$ in these areas (which is possible) in the case of volume integrals.

However, except for these formal differences, the formulation developed here follows Van der Vooren in the theoretical definition of viscous drag and wave drag. Indeed, this author stresses the fact that they are definitions ${ }^{6}$ : the result obtained in the particular case of a flow without trailing vorticity (and therefore without induced drag) is transposed to the general case of a flow with trailing vorticity and viscous drag plus wave drag is defined as the drag component expressed by the formula established in the particular case for total drag, i.e. with the present formulation,

$$
D_{v}+D_{w}=\iint_{S_{V W}}\left(\vec{f}_{v w}^{*} \cdot \vec{n}\right) d S
$$

\section{Eq.(13) is a result (in the absence of trailing vorticity). Eq.(14) is a definition.}

If it is further assumed that $\mathrm{Vv} \cap \mathbf{V w}=\varnothing$, and if $\mathrm{Sv}$ is the outer boundary of $\mathrm{Vv}$ (the inner boundary being $\left.\mathrm{S}_{\mathrm{A}}\right)$ and $\mathrm{Sw}$ the boundary of $\mathrm{Vw}(\mathrm{Vw}$, in the case of solutions to the RANS equations will be strictly included in the fluid, without contact with the body), see Figure 3, viscous drag and wave drag can be defined as

$$
\begin{aligned}
& D_{v}=\iint_{S_{V}}\left(\vec{f}_{v w}^{*} \cdot \vec{n}\right) d S \\
& D_{w}=\iint_{S_{W}}\left(\vec{f}_{v w}^{*} \cdot \vec{n}\right) d S
\end{aligned}
$$




\section{Definition of induced drag}

To derive an expression for induced drag, it is convenient to add to the definitions of $\vec{f}$ and $\vec{f}_{v w}^{*}$, eqs.(2),

(11), the definition of a third vector $\vec{f}_{i}^{*}$, such that

$$
\vec{f}=\vec{f}_{v w}^{*}+\vec{f}_{i}^{*}(\mathbf{1 7})
$$

i.e.

$$
\vec{f}_{i}^{*}=-\rho\left(u-u_{\infty}-\Delta \bar{u}\right) \vec{q}-\left(p-p_{\infty}\right) \vec{i}
$$

Eq.(6) is applicable to any closed surface So enveloping the aircraft. It is thus applicable to Svw, the outer boundary of the control volume $\mathrm{Vv} \cup \mathrm{Vw}$. Taking into account eq.(14), this equation leads to

$$
\begin{gathered}
D_{p}+D_{f}=\iint_{S_{V W}}(\vec{f} \cdot \vec{n}) d S \\
=\iint_{S_{V W}}\left(\vec{f}_{v w}^{*} \cdot \vec{n}\right) d S+\iint_{S_{V W}}\left(\vec{f}_{i}^{*} \cdot \vec{n}\right) d S \\
=D_{v}+D_{w}+\iint_{S_{V W}}\left(\vec{f}_{i}^{*} \cdot \vec{n}\right) d S
\end{gathered}
$$

The consequent definition of induced drag is the only one which will ensure an exact near-field / far-field drag balance given the previous definition of $D_{v}+D_{w}$, eq.(14). This expression has no obvious phenomenological meaning, contrary to the more familiar formulas involving the crossflow kinetic energy.

$$
\begin{gathered}
D_{i}=\iint_{S_{V W}}\left(\vec{f}_{i}^{*} \cdot \vec{n}\right) d S \text { (20) } \\
D_{p}+D_{f}=D_{v}+D_{w}+D_{i}
\end{gathered}
$$

\section{A one-vector formulation}

It is possible to eliminate vector $\vec{f}_{v w}^{*}$ in the formulation of the viscous and wave drag components, and thus to express all the far-field drag components with $\vec{f}_{i}^{*}$ alone. 
Let So be a closed surface in the far-field, strictly enclosing the control volumes Vv and Vw, as Su $\cup$ SL $\cup$ SD in Figure 3. Then, with the convention for normal vector orientation illustrated in the same figure, the zero divergence property of vector $\vec{f}$ (eq.(3)) allows to write

$$
\iint_{S_{0}}(\vec{f} \cdot \vec{n}) d S-\iint_{S_{V}}(\vec{f} \cdot \vec{n}) d S=0
$$

The first integral being equal to the total near-field drag (eq.(6)), and with the relation eq.(17) between $\vec{f}, \vec{f}_{v w}^{*}$ and $\vec{f}_{i}^{*}$, eq.(22) may be rewritten as

$$
D_{p}+D_{f}=\iint_{S_{V}}\left(\vec{f}_{v w}^{*} \cdot \vec{n}\right) d S+\iint_{S_{V}}\left(\vec{f}_{i}^{*} \cdot \vec{n}\right) d S
$$

and, with the definition of $\mathrm{D}_{\mathrm{v}}$, eq.(15),

$$
D_{v}=D_{p}+D_{f}-\iint_{S_{V}}\left(\vec{f}_{i}^{*} \cdot \vec{n}\right) d S
$$

or, $\mathrm{D}_{\mathrm{vp}}=\mathrm{D}_{\mathrm{v}}-\mathrm{D}$, denoting the viscous pressure drag.

$$
D_{v p}=D_{p}-\iint_{S_{V}}\left(\vec{f}_{i}^{*} \cdot \vec{n}\right) d S
$$

Similarly, using surfaces So, Sw, SA and the definition of wave drag eq.(16), the following expression can be derived for $\mathrm{D}_{\mathrm{w}}$ :

$$
D_{w}=-\iint_{S_{W}}\left(\vec{f}_{i}^{*} \cdot \vec{n}\right) d S
$$

Equations (24), (25), (26) constitute a one-vector formulation for the far-field drag components. The main motivation for the choice $\vec{f}_{v w}^{*}$ instead of $\vec{f}_{v w}$ earlier in the theory is the economy of the computation of $\vec{\tau}_{x}$ for the one-vector pressure drag breakdown, and the fact of having to compute it only on the aircraft surface for the total drag breakdown. In this formulation, $\vec{\tau}_{x}$ being absent in the expression of $\vec{f}_{i}^{*}$, friction stresses do not appear in the far-field integrals in eqs.(20), (24), (25) and (26), only in the global friction drag term in eq.(24). This formulation basically gives a breakdown of pressure drag: 


$$
D_{p}=D_{v p}+D_{w}+D_{i}
$$

\section{Definition of spurious drag}

Spurious drag is defined as drag produced through entropy or stagnation enthalpy variation along streamlines outside physical viscous layers and shocks, and not resulting from vortex decay. If VSP is that part of the computational volume outside $\mathrm{Vv}_{\mathrm{v}} \cup \mathrm{Vw}$ in which such phenomena occur, since by definition $\mathrm{VsP} \cap \mathrm{Vv}=\varnothing$ and since the body is completely immerged in the fluid contained in Vv, the boundary SsP of VsP will not intersect SA. Then, spurious drag can be expressed in a similar way to wave drag, eq.(26),

$$
D_{s p}=-\iint_{S_{S P}}\left(\vec{f}_{i}^{*} \cdot \vec{n}\right) d S
$$

The line of reasoning which led in the previous section to the theoretical near-field/far-field drag balances, eqs.(21), (27), then gives the following numerical drag balances,

$$
\begin{gathered}
D_{p}+D_{f}=D_{v}+D_{w}+D_{i}+D_{s p} \\
D_{p}=D_{v p}+D_{w}+D_{i}+D_{s p}
\end{gathered}
$$

The splitting of vector $\vec{f}$ into $\vec{f}_{i}^{*}$ and $\vec{f}_{v w}^{*}$ instead of $\vec{f}_{i}$ and $\vec{f}_{v w}$ makes it clear that spurious drag is a component of pressure drag, eq.(30).

\section{Definition of induced drag in the presence of spurious drag}

In the presence of spurious drag, the expressions for viscous drag, viscous pressure drag and wave drag, eqs.(24), (25), (26) remain unchanged, but the expression for induced drag must be modified.

The theoretical definition of induced drag, eq.(20), comes from the expression of total drag as the integral of the quantity $(\vec{f} . \vec{n}) \mathrm{dS}$ over Svw, see eq.(19) :

$$
D=\iint_{S_{V W}}(\vec{f} \cdot \vec{n}) d S=D_{v}+D_{w}+\iint_{S_{V W}}\left(\vec{f}_{i}^{*} \cdot \vec{n}\right) d S
$$


To obtain a definition of induced drag compatible with the modified drag balance, eq.(29), the surface for the integration of $(\vec{f} \cdot \vec{n}) \mathrm{dS}$ must be $\mathrm{Svw} \cup$ Ssp instead of Svw,

$$
D=\iint_{S_{V W} \cup S_{S P}}(\vec{f} \cdot \vec{n}) d S=D_{v}+D_{w}+D_{s p}+\iint_{S_{V W} \cup S_{S P}}\left(\vec{f}_{i}^{*} \cdot \vec{n}\right) d S
$$

which is allowed by the fact that the surface remains a closed surface enveloping the body, see eq.(6).

The modified definition of induced drag in the presence of spurious drag is then

$$
D_{i}=\iint_{S_{V W} \cup S_{S P}}\left(\vec{f}_{i}^{*} \cdot \vec{n}\right) d S
$$

\section{Corrected far-field drag}

A corrected far-field drag formula is straightforwardly obtained by suppressing the spurious drag component in eq.(29),

$$
D_{f f, c o r r}=D_{v}+D_{w}+D_{i}(\mathbf{3 4})
$$

and

$$
D_{f f, c o r r}=D_{f f}-D_{s p}=D_{n f}-D_{s p}(\mathbf{3 5})
$$

$\mathrm{D}_{\mathrm{v}}, \mathrm{D}_{\mathrm{w}}, \mathrm{D}_{\mathrm{i}}$ being obtained from eqs.(24), (26), (33).

This is an advantage of the far-field drag approach: it not only allows to obtain a physical breakdown of drag, but also to eliminate the spurious drag component. It is obvious from eq.(29) that such elimination is absolutely impossible in the near-field.

In practice, the control volumes $\mathrm{Vv}$ and $\mathrm{Vw}$ are not defined manually, but automatically, using physical criteria $^{9,10,11}$, a viscous layer detector and a shock detector. The reliability of the viscous and wave drag component extraction will depend on the reliability of these detectors, and on the ability of the flow solver to damp artificial dissipation in the physical viscous layers. 
The computation of $\mathrm{Di}_{\mathrm{i}}$ also requires the definition of VSP (see eq.(33)). In the absence of trailing vorticity artificial decay, VsP is that part of the computational domain outside $\mathrm{Vv} \cup \mathrm{Vw}$ where there is production (or destruction) of vector $-\vec{f}_{i}^{*}$. But this quantity is not smooth enough to be a reliable sensor.

Since outside $V_{V} \cup V_{w} \cup$ VsP production/destruction of $-\vec{f}_{i}^{*}$ is zero, and considering eq.(33), VsP may be identified with the entire computational volume $\mathrm{V}$ minus $\mathrm{Vv} \cup \mathrm{Vw}$, and $\mathrm{Svw} \cup \mathrm{SsP}$ replaced by the outer boundary of $\mathrm{V} \partial \mathrm{ext} \mathrm{V}$ in this equation. But this is only correct in the absence of trailing vortex spurious decay.

\section{Consequences of trailing vortex artificial decay}

With trailing vortex artificial decay, "apparent" induced drag decreases as the downstream boundary of the control volume moves downstream, and may in some cases reach zero before the downstream boundary reaches the boundary of the computational domain ${ }^{12}$.

A correction method was proposed in reference ${ }^{13}$, based on the idea that spurious vortex diffusion being an irreversible process, the lost induced drag must reappear as a kind of spurious viscous drag.

Corrected induced drag is obtained by adding this quantity to the apparent induced drag,

$$
D_{i, c o r r}=D_{i, a p p}+D_{\text {diff }}
$$

With the one-vector formulation considered here, this technique takes a particular form. If VTV is a volume containing the trailing vortices, the correction is expressed as spurious drag, eq.(28),

$$
D_{\text {diff }}=-\iint_{S_{T V}}\left(\vec{f}_{i}^{*} \cdot \vec{n}\right) d S
$$

where STV is the boundary of VTV . So, if $\partial_{\mathrm{ext}} \mathrm{V}$ is the outer boundary of the computational domain, eq.(36) becomes

$$
D_{i, c o r r}=\iint_{\partial_{e x t} V}\left(\vec{f}_{i}^{*} \cdot \vec{n}\right) d S-\iint_{S_{T V}}\left(\vec{f}_{i}^{*} \cdot \vec{n}\right) d S=\iint_{S_{I}}\left(\vec{f}_{i}^{*} \cdot \vec{n}\right) d S
$$


where $\mathrm{SI}_{\mathrm{I}}$ is the outer boundary of the control volume Vi which is currently defined as shown in the Figure 4 . The lift induced drag coefficient is computed thanks to this equation. It is a paradox of the one-vector formulation, that the trailing vortices, which contain the crossflow kinetic energy related to induced drag, must be excluded from this control volume.

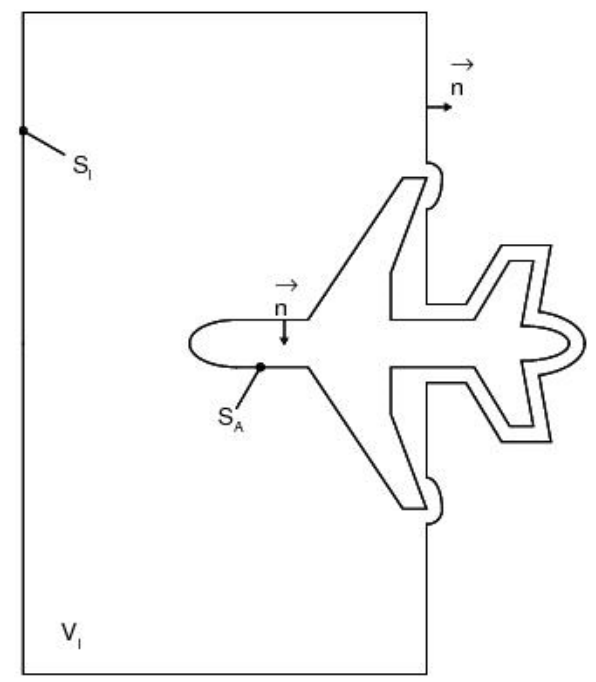

Figure 4 : Control surfaces and volume for far-field extraction of induced drag

In this study, far-field analysis are carried out thanks to the drag extraction software ONERA-ffd72 $2^{6,12,14}$. It is based on the formulations and methods presented above, in particular, the coefficient CDff used below corresponds to $D_{f f, c o r r}$ (eq.(34)) . This software is developed to provide a physical drag breakdown into viscous, wave and lift-induced drags and therefore to eliminate spurious drag by difference with the sum of pressure and friction drag coefficients. It also permits to locate the different drag productions (control volumes). These capacities are very helpful for aircraft manufacturers.

\section{Grid convergence study}

The grid convergence study corresponds to Test Case 1.1 of the $4^{\text {th }}$ Drag Prediction Workshop and concerns the wing/body/horizontal tail configuration with tail incidence angle $\mathrm{iH}=0.0^{\circ}$. The aerodynamic conditions are the following:

- $\quad$ Mach number: $\mathrm{M}=0.85$; 
- Lift coefficient $\mathrm{CL}=0.500( \pm 0.001)$;

- Reynolds number Re/c $=5.10^{6}$ corresponding to NASA-Ames $11-\mathrm{ft}$ transonic wind tunnel conditions.

The four available multi-block Boeing grids presented in the previous section (see Table 1) are used for this study: the coarse, the medium, the medium-fine and the fine grids. In order to ensure a high convergence level, 1,800 iterations were done for all the computations. Figure 5 shows a comparison of the lift and total drag convergence obtained with the 4 grid refinements.

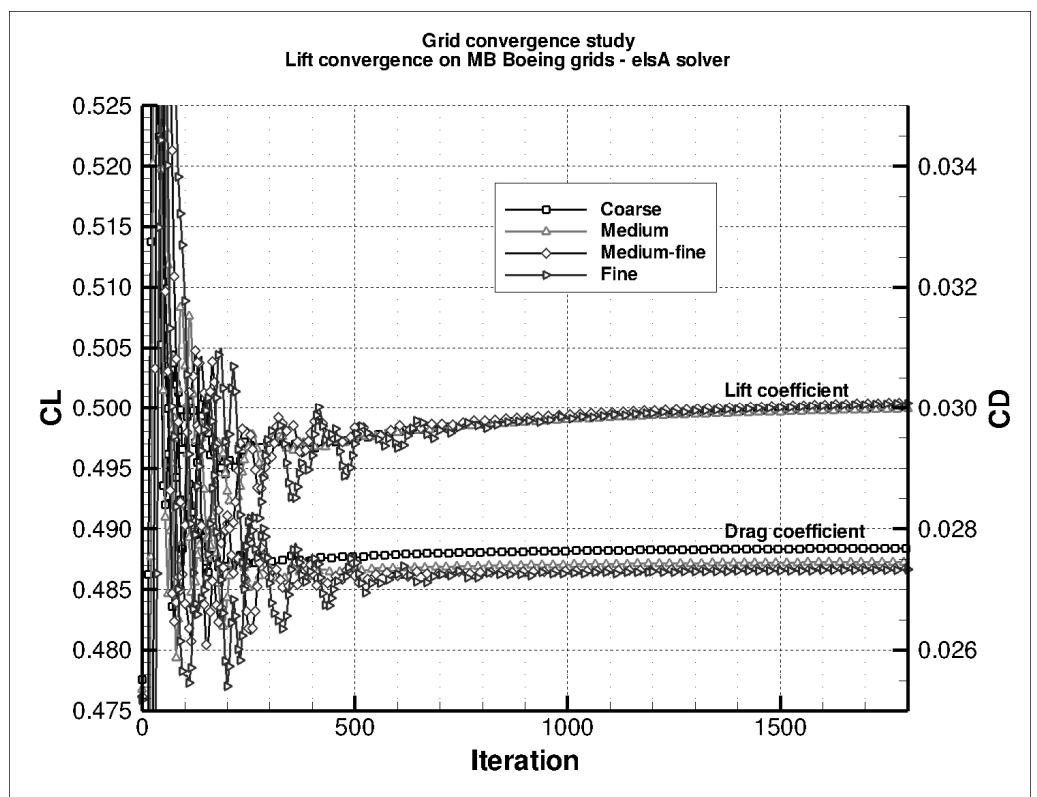

Figure 5. CRM wing / body / horizontal tail plane configuration $\left(\mathrm{iH}=0.0^{\circ}\right)$ - Grid convergence study - Lift and drag convergence.

\section{A. Drag and pitching moment predictions}

The grid convergence study is summarized in Table 2 and Table 3 where angles of attack, near-field drag values (direct integration of pressure and friction forces at the aircraft surface), as well as the pitching-moment, obtained with the elsA code are presented. Far-field drag coefficients given by the ffd 72 code are also available. All the drag coefficients are given in drag counts $\left(1\right.$ count $\left.=10^{-4}\right)$.

Table 2. CRM WBH configuration $\left(\mathrm{iH}=\mathbf{0 . 0 ^ { \circ }}\right)$ - Grid convergence study $\left(\mathrm{M}=\mathbf{0 . 8 5}, \mathrm{Re} / \mathrm{c}=5.10^{6}, \mathrm{CL}=\mathbf{0 . 5 0}\right)-$ Lift and pitching moment coefficients.

\begin{tabular}{|c|c|c|c|c|c|}
\hline CRM tail (iH =0.0 $^{\circ}$ ) & $\boldsymbol{\alpha}$ & CL & CLwing & CLhtp & CM \\
\hline Coarse grid & 2.35 & 0.5000 & 0.4552 & -0.0158 & -0.0487 \\
\hline Medium grid & 2.34 & 0.4999 & 0.4554 & -0.0161 & -0.0471 \\
\hline
\end{tabular}




\begin{tabular}{|c|c|c|c|c|c|}
\hline Medium fine grid & 2.35 & 0.5005 & 0.4741 & -0.0163 & -0.0457 \\
\hline Fine grid & 2.36 & 0.5004 & 0.4742 & -0.0164 & -0.0444 \\
\hline
\end{tabular}

Table 3. CRM WBH configuration $\left(\mathrm{iH}=\mathbf{0 . 0 ^ { \circ }}\right)$ - Grid convergence study $\left(\mathrm{M}=\mathbf{0 . 8 5}, \mathrm{Re} / \mathrm{c}=5.10^{6}, \mathbf{C L}=\mathbf{0 . 5 0}\right)-$ Near-field and far-field drag coefficients.

\begin{tabular}{|c|c|c|c|c|c|c|c|c|c|c|}
\hline CRM tail (iH =0.0 $^{\circ}$ ) & CL & CDp & CDf & CDnf & CDvp & CDv & CDi & CDw & CDff & CDsp \\
\hline Coarse grid & 0.5000 & 147.6 & 129.2 & 276.8 & 47.6 & 176.8 & 92.4 & 5.9 & 275.1 & 1.7 \\
\hline Medium grid & 0.4999 & 146.0 & 128.6 & 274.6 & 46.7 & 175.3 & 92.3 & 6.0 & 273.5 & 1.0 \\
\hline Medium fine grid & 0.5005 & 145.6 & 127.9 & 273.5 & 46.1 & 174.0 & 92.5 & 6.3 & 272.8 & 0.7 \\
\hline Fine grid & 0.5004 & 145.6 & 127.6 & 273.2 & 46.0 & 173.6 & 92.6 & 6.5 & 272.7 & 0.5 \\
\hline
\end{tabular}

Keeping in mind that a high level of precision is required in this study, we can notice:

- A logical decrease of the pressure drag value of 2 drag counts with the increase of mesh refinement. This is due to a better discretisation of the computational domain which leads to a more accurate solution and a weaker artificial drag coefficient (CDsp);

- For the friction drag, a 1.6 drag count decrease is observed from the coarse to the fine grid noting that the $y+$ is reduced with the mesh refinement increase;

- The refinement has also a non negligible impact on the pitching-moment prediction with a range from -0.0487 on the coarse grid to -0.0444 on the fine grid. It is interesting to notice a pitching moment variation between the medium fine and the fine grid though the wing lift and the tail lift are quite the same for these computations. The pitching-moment component appears to be a very sensitive component to evaluate. Remark: it will be very useful in the future to define a level of precision for the pitching-moment prediction in order to evaluate the quality of the results.

The lift and drag values obtained respectively with the medium-fine grid computation (26.0 million nodes) and the fine grid computation (47.8 million nodes) are in very good agreement. Indeed these two computations provide:

- Quite the same pressure drag value;

- 0.3 drag count of difference on the friction drag component;

- A scatter of 0.0013 point on the pitching-moment coefficient. 
The previous comments are explicitly illustrated in Figure 6. Pressure and friction drag components seem to tend asymptotically to a given value while the pitching-moment does not seem to converge as rapidly as the drag component till a given value.

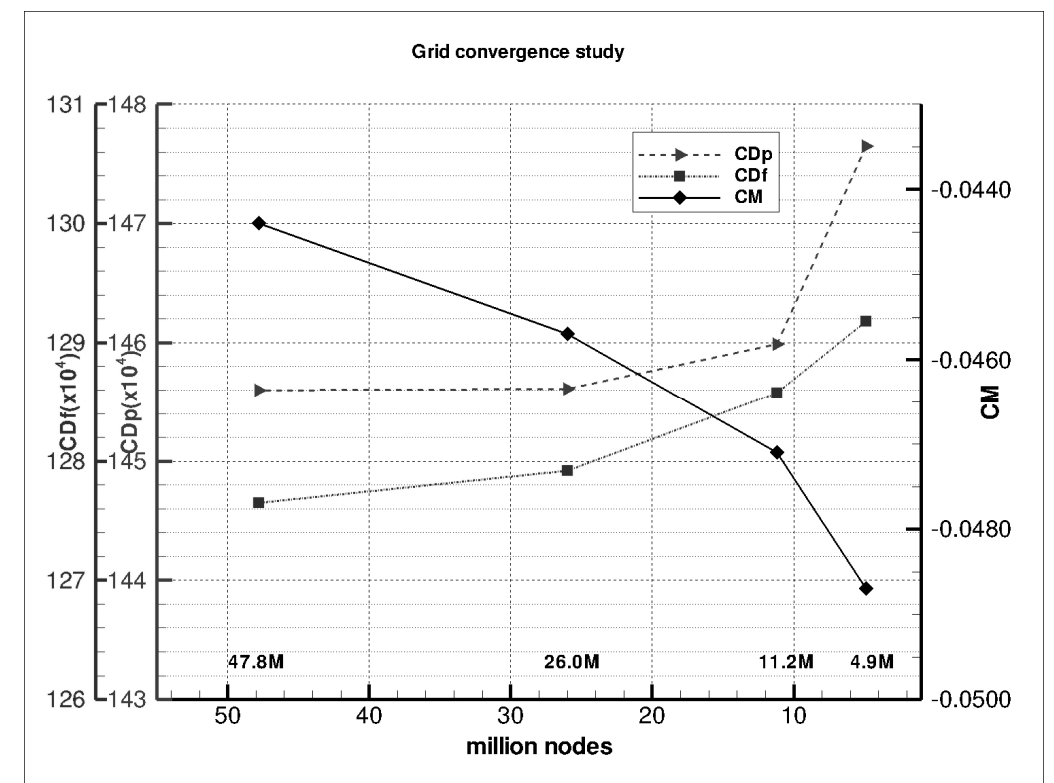

Figure 6. CRM wing / body / horizontal tail plane configuration $\left(\mathrm{iH}=0.0^{\circ}\right)$ - Grid convergence study Pressure drag, friction drag and pitching-moment v.s mesh refinement.

The far-field drag values are presented in Table 3, the grid convergence study shows that:

- The grid refinement has a non negligible impact on the viscous drag component. This scatter comes respectively from the friction drag component and from the viscous pressure drag component (flow separation, wake...). With the different grid levels, different flow phenomena are captured, for instance, there is no flow separation at the wing-trailing-edge / body junction with the coarse grid while a small flow separation is captured with the finest one. Concerning the viscous drag scatter between the medium-fine and the fine results; it is very reduced and reveals a really good convergence with these very well refined grids;

- Whatever the grid level is, a very good agreement is obtained on the lift-induced drag component;

- A limited impact is observed on the wave drag component from the coarse to the fine grid with a variation of 0.6 drag count;

- Concerning the spurious drag, it can be noticed that the values are very low, even for the coarse grid. It demonstrates the really good quality of these grids. 
As a partial conclusion, this grid convergence study exhibits a drag variation of around 3 drag counts when including the coarse grid and a variation limited to around 1 drag count when starting the grid convergence study from the medium grid. A real convergence effect is observed on the drag components. The same argument is not valid for the pitching moment evaluation because even if the coarse grid provides the highest pitching moment value, no real convergence till a given value is observed for this component with the grid refinement increase.

\section{B. ONERA and other participants results}

First, a comparison with the Boeing results on the same grids is given. Boeing performed full Navier-Stokes and thin layer computations with CFL3D code on the provided multi-block structured grids ${ }^{15}$. The drag and pitchingmoment values from ONERA are added to the Boeing slides presented in June 2009 to the DPW4 community. This very interesting comparison between Onera and Boeing results is presented in Figure 7 and Figure 8.
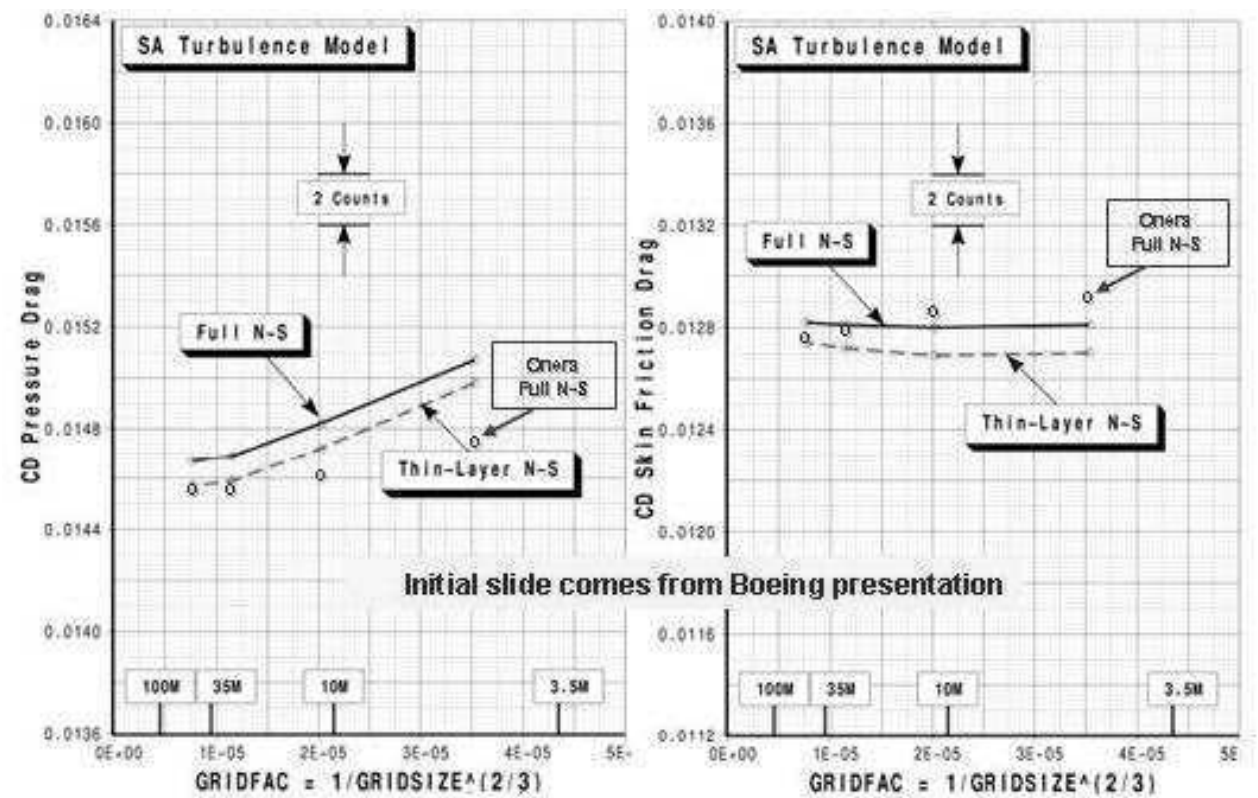

Figure 7. CRM wing / body / horizontal tail plane configuration $\left(\mathrm{iH}=0.0^{\circ}\right.$ ) - Grid convergence study - Onera v.s Boeing results on the same multiblock grids: pressure and friction drags.

A good agreement is obtained on the total drag values: the ONERA results are very similar to the Boeing thin layer values and less than 2 drag counts different from the Boeing full Navier-Stokes computations.

The breakdown in pressure and skin friction drags shows that: 
- The pressure drag is slightly less dissipative for the ONERA computations, it tends to the same value as the Boeing thin layer computation and is less than 1 drag count far from the Boeing full NS results;

- Concerning the skin friction drag, Boeing results are less dependent to the grid refinement and the scatter between ONERA and Boeing is lower than 2 drag counts.

For the pitching-moment prediction, the ONERA value on the fine grid is between and very close to the Boeing full Navier-Stokes and thin layer predictions. For a first comparison of pitching-moment evaluation, this result is quite satisfying.

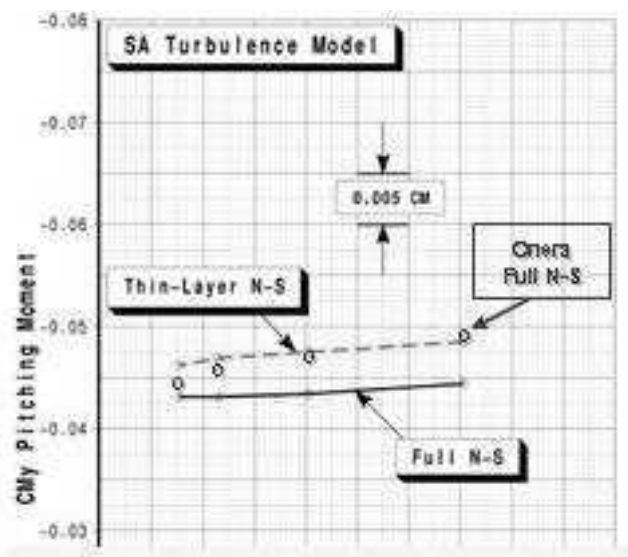

Initial slide comes from Boeing presentation

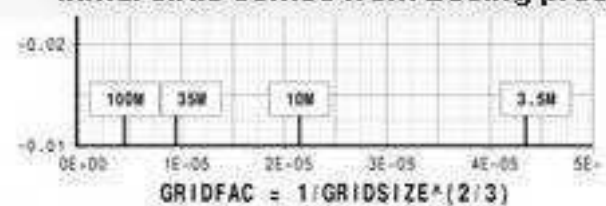

Figure 8. CRM wing / body / horizontal tail plane configuration $\left(\mathrm{iH}=0.0^{\circ}\right)$ - Grid convergence study - Onera v.s Boeing results on the same multiblock grids: pitching moment.

Using the results given in the Summary of the fourth AIAA CFD Drag Prediction Workshop ${ }^{16}$, a comparison with the other participants' results on medium grids is proposed here. The computations of Boeing (B), Airbus (A), DLR (D) and ONERA (O) are highlighted. Concerning the total drag (Figure 9), the results from these 4 participants are close to the NTF experimental value of 275 drag counts. For the pressure drag (Figure 10), the structured computations show a scatter of less than 2 d.c. Concerning the friction drag coefficient (Figure 11), the values pushed forward here are higher of about 5d.c than the CFD average. The pitching moment predictions of Boeing, Airbus, DLR and ONERA reveal very good agreement and stick to the CFD average (Figure 12). These 
drag and moment predictions are very interesting and the agreement which is achieved between these 4 participants of equivalent maturity is quite satisfying.

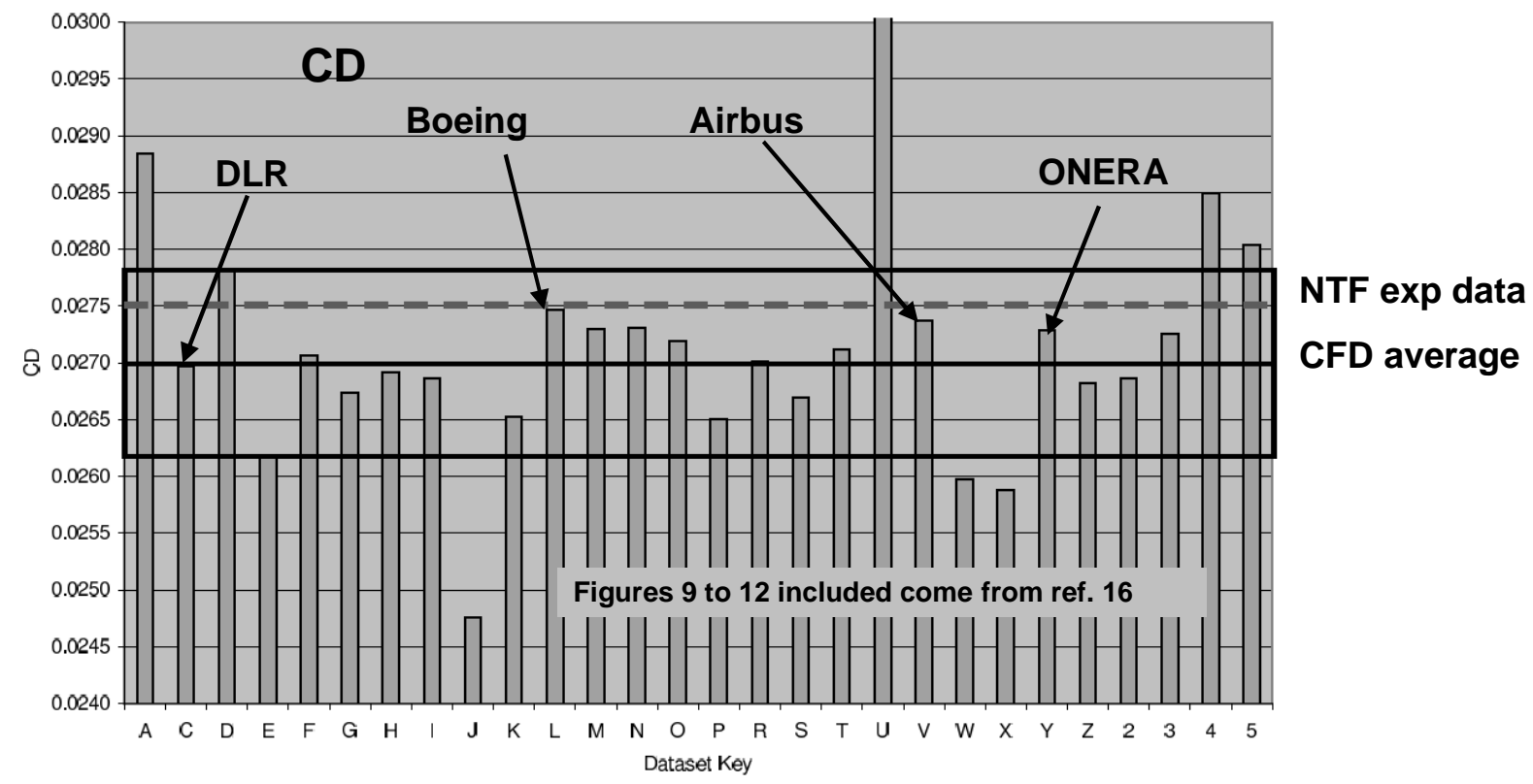

Figure 6. Case 1a Continuum Estimates of Total Drag: $M=0.85, C_{L}=0.5, R e=5$ million.

Figure 9. CRM wing / body / horizontal tail plane configuration $\left(\mathrm{iH}=0.0^{\circ}\right)$ - Grid convergence study - Onera and other participants: total drag.

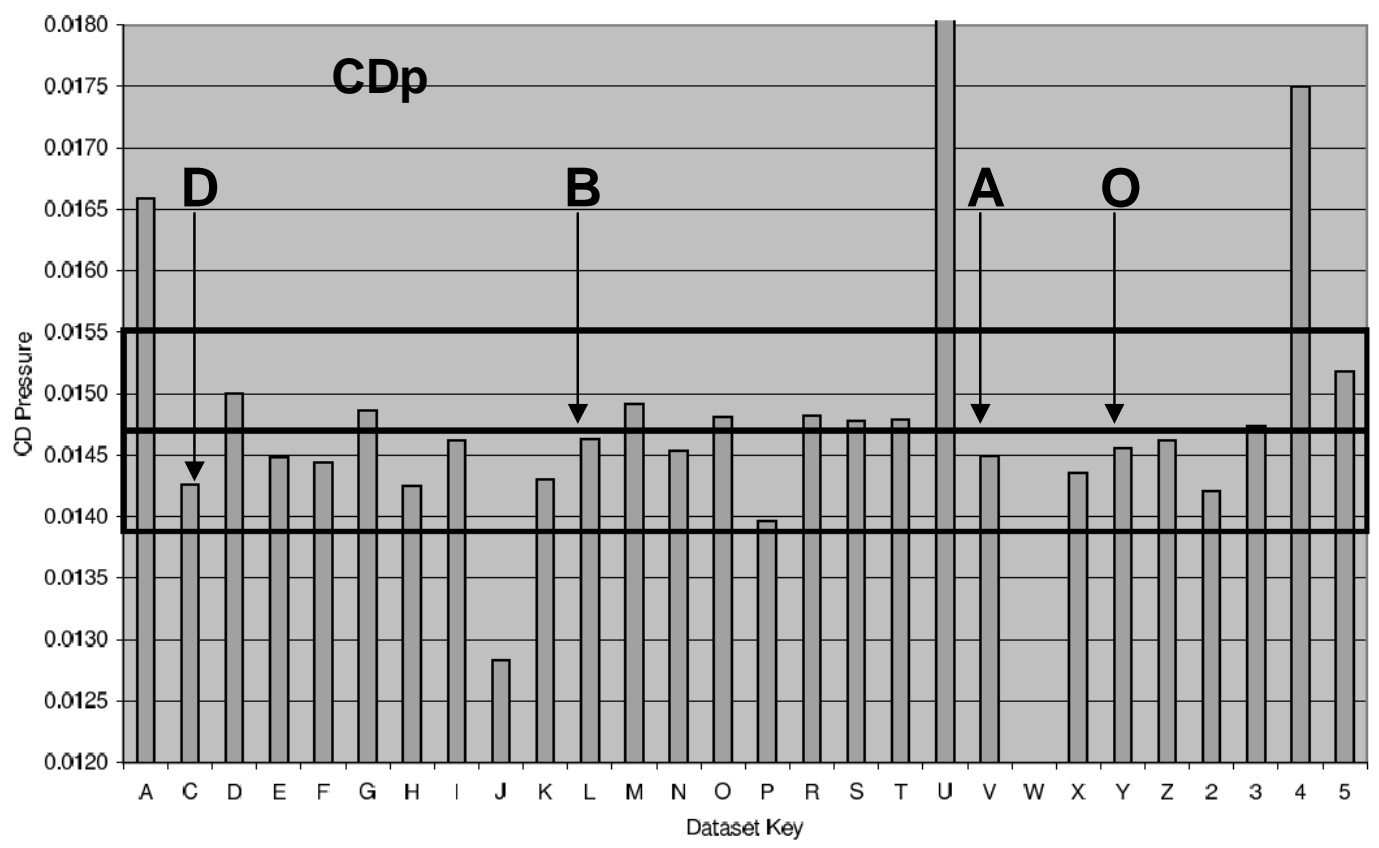

Figure 8. Case 1a Continuum Estimates of Pressure Drag: $M=0.85, C_{L}=0.5, R e=5$ million.

Figure 10. CRM wing / body / horizontal tail plane configuration $\left(\mathrm{iH}=0.0^{\circ}\right)$ - Grid convergence study - Onera and other participants: pressure drag. 


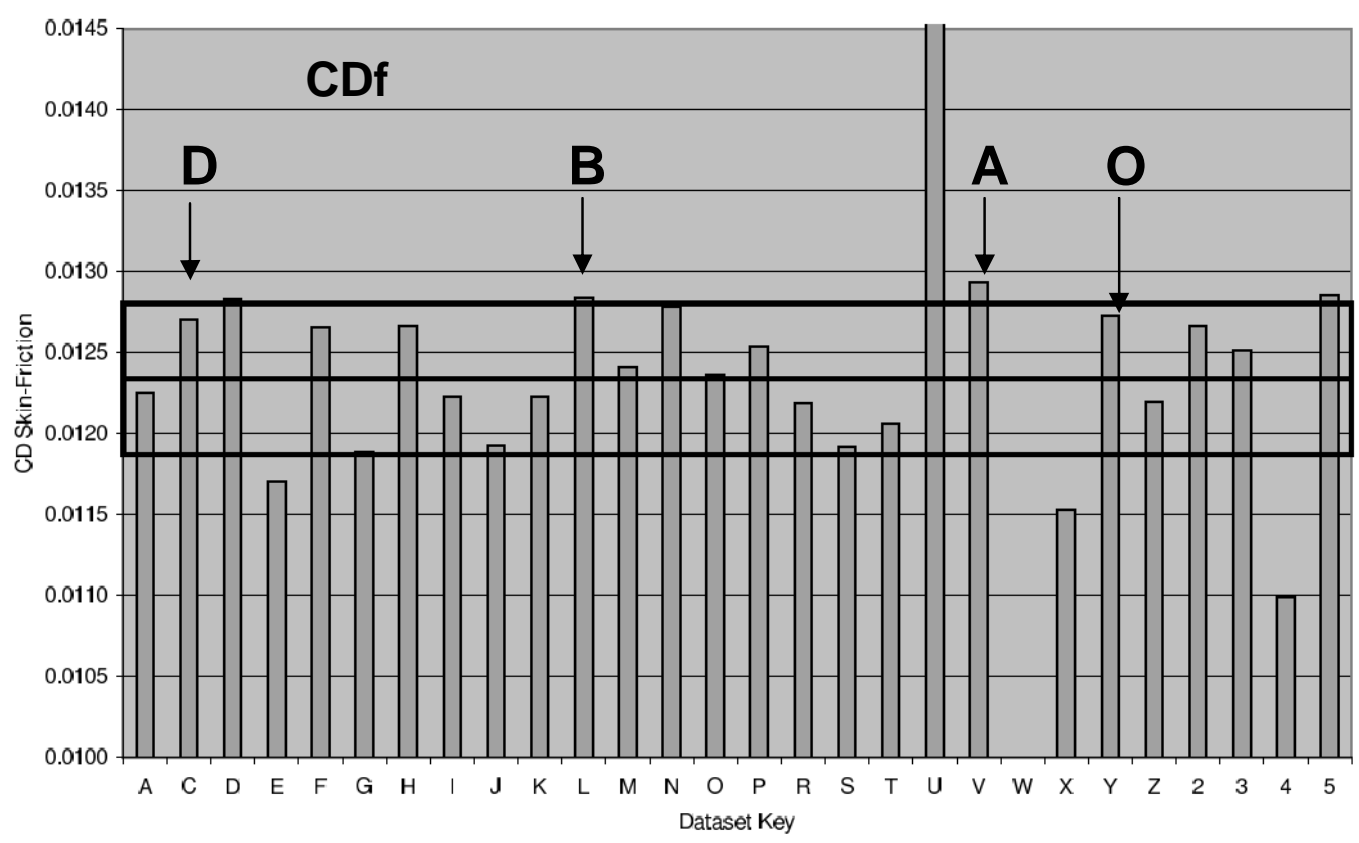

Figure 10. Case 1a Continuum Estimates of Skin-Friction Drag: $M=0.85, C_{L}=0.5, R e=5$ million.

Figure 11. CRM wing / body / horizontal tail plane configuration $\left(\mathrm{iH}=0.0^{\circ}\right)$ - Grid convergence study - Onera and other participants: friction drag.

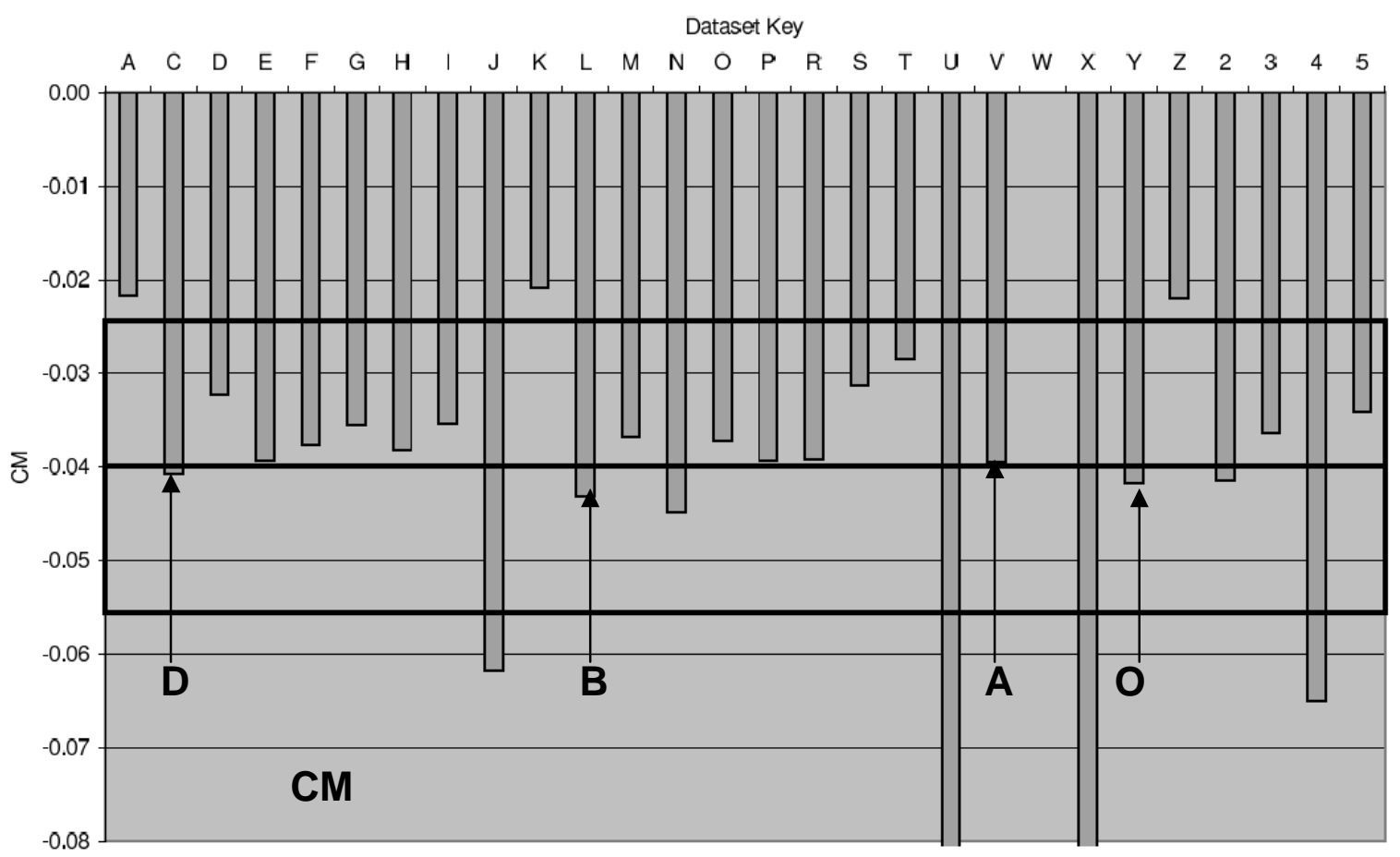

Figure 12. Case 1a Continuum Estimates of Pitching-Moment: $M=0.85, C_{L}=0.5, R e=5$ million.

Figure 12. CRM wing / body / horizontal tail plane configuration $\left(\mathrm{iH}=0.0^{\circ}\right)$ - Grid convergence study - Onera and other participants: pitching moment. 


\section{Downwash study}

\section{A. Computations and results}

The downwash study corresponds to Test Case 1.2 of the DPW4. It concerns 4 different configurations: the wing/body/tail-off and the wing/body/horizontal-tail with 3 tail deflection angles $\left(0^{\circ},-2^{\circ}\right.$ and $\left.+2^{\circ}\right)$. This downwash study is realized on the medium grid in the following conditions:

- $\quad$ Mach number: $\mathrm{Ma}=0.85$;

- Drag polars for $\alpha=0.0^{\circ}, 1.0^{\circ}, 1.5^{\circ}, 2.0^{\circ}, 2.5^{\circ}, 3.0^{\circ}, 3.5^{\circ}, 4.0^{\circ}$;

- $\quad$ Tail deflection angles $0^{\circ},-2^{\circ},+2^{\circ}$ and tail-off;

- $\quad$ Reynolds number $\mathrm{Re} / \mathrm{c}=5.10^{6}$ corresponding to NASA-Ames 11-ft transonic wind tunnel conditions.

In order to ensure a high convergence level, 1,800 iterations were done for all the computations as in the previous "Grid Convergence study". The computations have also been carried out at CL=0.50 in order to provide an additive reference value and to permit a straightforward comparison of the 4 configurations.

The following figures present the results obtained on the 4 configurations through lift, drag and pitching moment polars.

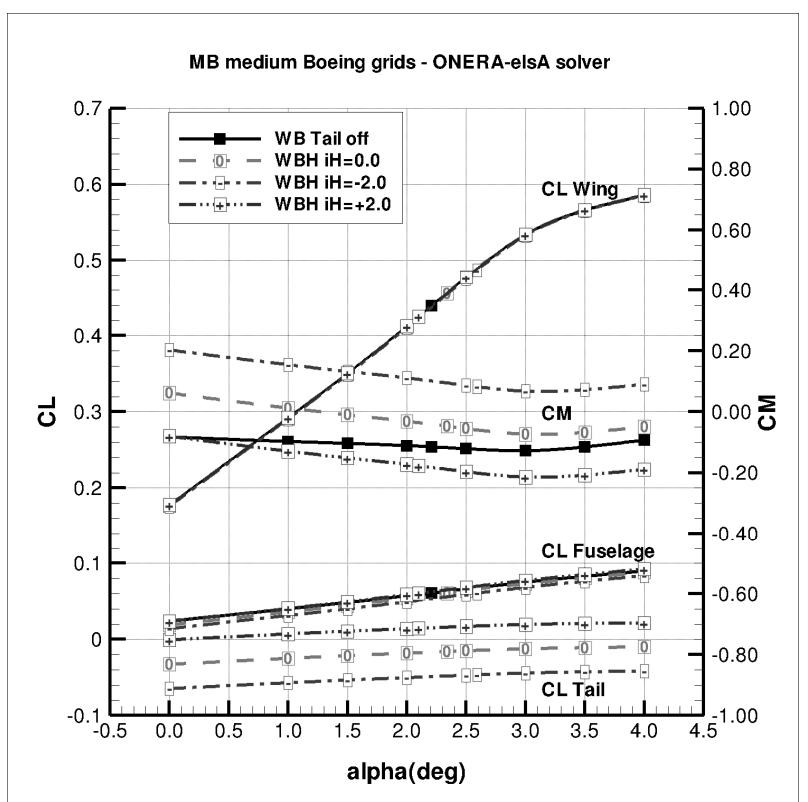

Figure 13. CRM wing / body / horizontal tail plane configuration - Downwash study - Lift polars of the aircraft components and corresponding pitching moment values. 
Figure 13 shows the lift polars part by part: wing, fuselage and tail, as well as the corresponding global pitching moments for the 4 above-mentioned configurations. The wing lift coefficient is independent of the tail configuration. The fuselage CL exhibits a light variation when the empennage setting is modified, it reveals an HTP/body interaction. The main discrepancies between configurations when looking global CL is therefore due to the tail plane lift contribution which is obviously strongly dependent on the deflection angle, the empennage showing quite a symmetric profile.

On the wing/body/tail-off configuration, for each angle of attack the pitching moment is negative. It means that the lift produced by the wing leads to a counter-clockwise rotation (if the configuration is seen as it is on the second illustration of Figure 1) about the center of gravity of the airplane. So, in this case, only a negative tail lift producing a clockwise rotation would allow the aircraft to be trimmed.

Figure 13 points out that for the empennage setting $\mathrm{iH}=0.0^{\circ}$ the tail lift is negative for all the computed angles of attack (downwash effect). It then produces a clockwise rotation about the center of gravity which can balance the natural counter-clockwise wing rotation moment. It is important to notice that, for the angles of attack from $0.0^{\circ}$ to $4.0^{\circ}$, the pitching-moment goes from a positive value to a negative value. The empennage setting iH $=-2.0^{\circ}$ generates a logical stronger negative tail lift which leads for each angle of attack to a positive pitching moment. So this tail deflection is too negative to obtain the target $\mathrm{CM}=0$. With the third empennage setting $\mathrm{iH}=+2.0^{\circ}$, the tail lift is slightly negative at $0^{\circ}$ angle of attack and positive for all the other angles of attack. This tail incidence generates a counter-clockwise rotation which does not counter the wing rotation moment. 


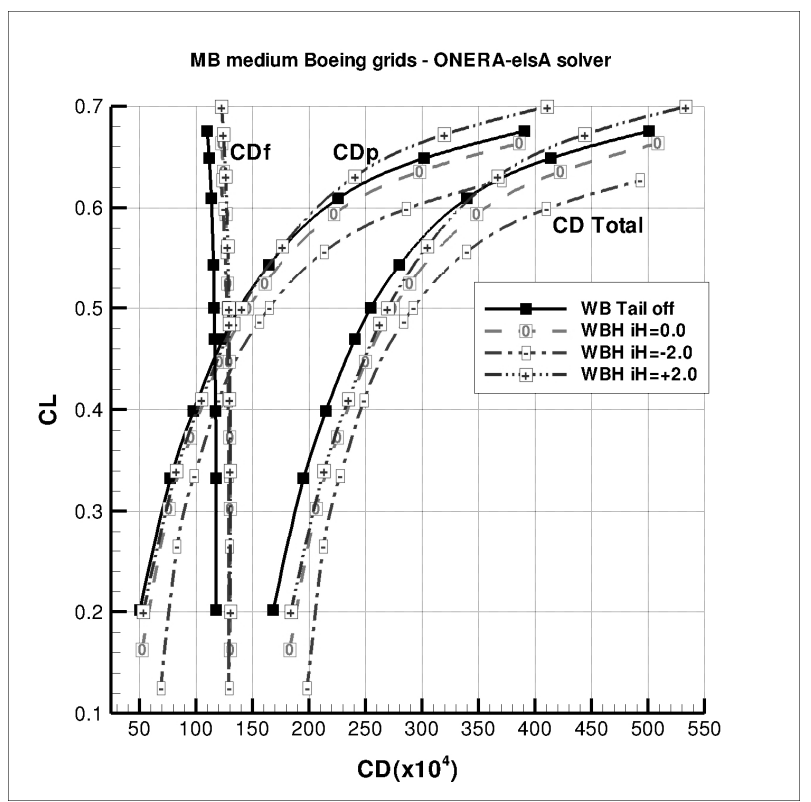

Figure 14. CRM wing / body / horizontal tail plane configuration - Downwash study - Pressure drag, skin friction drag and total drag for different lift values.

Figure 14 highlights the tail incidence effects on the near-field drag coefficients. First, the skin friction drag is naturally impacted by the addition of the wetted surface area of the empennage. It represents a penalty of 11 to 13 drag counts between the tail-off and tail-on configurations. The CDf penalty is almost independent of the tail incidence. On the contrary, the pressure drag is strongly dependent on the tail incidence, for instance at CL $=0.5$, the pressure drag penalty is about 7 counts for $\mathrm{iH}=0.0^{\circ}$ and about 26 counts for $\mathrm{iH}=-2.0^{\circ}$.

Figure 15. CRM wing / body / horizontal tail plane configuration $\left(\mathrm{iH}=0.0^{\circ}\right)-$ Medium grid $(\mathrm{CL}=0.50, \alpha=$ $2.34^{\circ}$ ) - Downwash effect visible by streamlines on the wing and on the horizontal tail.

As a complementary illustration, the downwash effect of the wing on the configuration at $\mathrm{CL}=0.50, \alpha=2.34^{\circ}$, $\mathrm{Re} / \mathrm{m}=5.10^{6}$ with empennage setting $\mathrm{iH}=0.0^{\circ}$ is shown using streamlines in Figure 15 . Because of the downwash effect, the apparent angle of attack for the empennage is negative and explains the negative tail lift value (see Figure 13) in these conditions. 


\section{B. Trim drag evaluation}

The pitching-moment values are plotted in Figure 16 for the 4 configurations. Figure 16 and Figure 17 show that the required empennage setting to trim the aircraft at $\mathrm{CL}=0.5$ will be between $\mathrm{iH}=0.0^{\circ}$ and $\mathrm{iH}=-2.0^{\circ}$. Through a linear interpolation on the curve $\mathrm{CL}(\mathrm{CM})$, the empennage setting which can trim the configuration $(\mathrm{CM}=0)$ is evaluated. As a result, for the target lift coefficient value $\mathrm{CL}=0.50$, the aircraft should be trimmed with an empennage incidence $\mathrm{iH}=-0.72^{\circ}$.

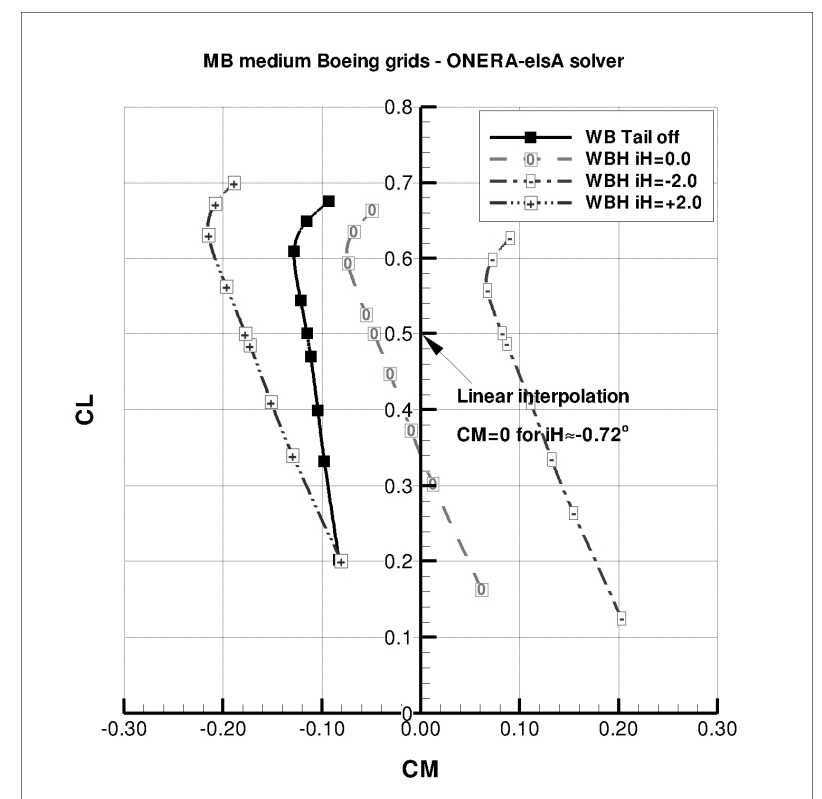

Figure 16. CRM wing / body / horizontal tail plane configuration - Downwash study - CL(CM). 


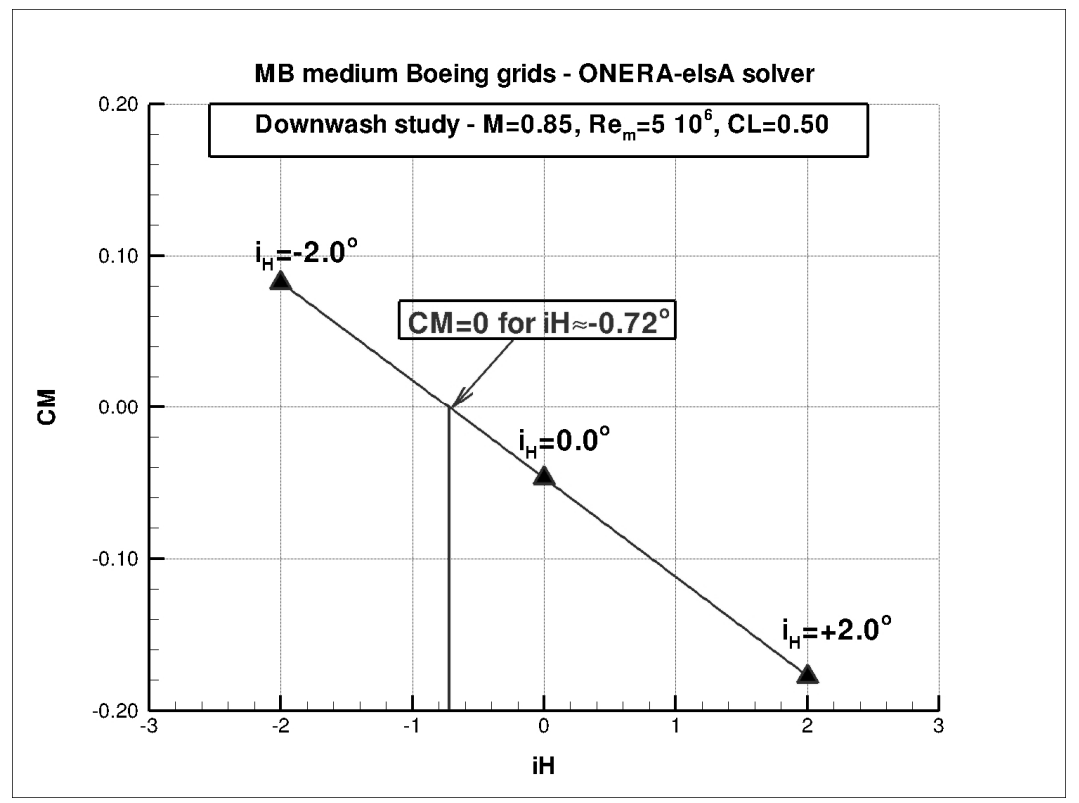

Figure 17. CRM wing / body / horizontal tail plane configuration - Downwash study - Evaluation of the trimming HTP deflection angle at $\mathrm{CL}=0.5$.

Figure 18 illustrates the trim drag penalty concept by showing the impact of tail trimming on the near-field drag components, specifically for $\mathrm{CL}=0.50$. The trim drag is here defined as the difference between the tail-off configuration drag and the trimmed configuration drag at the same lift. For CL $=0.50$, the trim drag can be evaluated as the difference between the tail-off configuration and the interpolated $\mathrm{iH}=-0.72^{\circ}$ configuration.

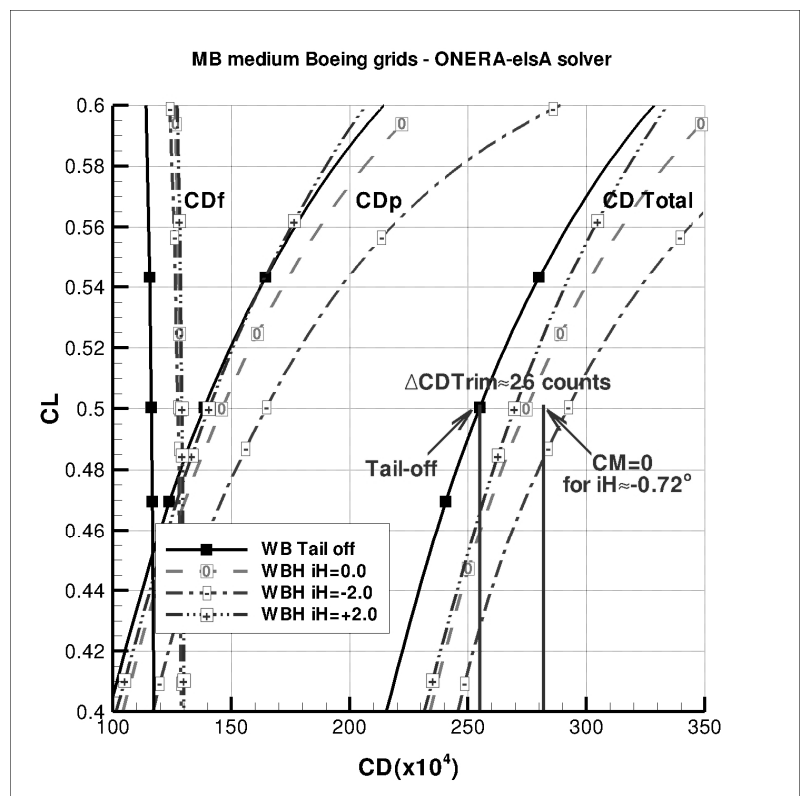

Figure 18. CRM wing / body / horizontal tail plane configuration - Downwash study - Evaluation of trim drag value. 
Knowing that the trimmed configuration should be obtained for an empennage setting around $-0.72^{\circ}$ and following an approximate linear drag increment (between $\mathrm{iH}=0.0^{\circ}$ and $\mathrm{iH}=-2.0^{\circ}$ configurations), it is possible to evaluate the drag penalty for the trimmed configuration.

Table 4 and Table 5 give the drag and pitching moment values for the tail-off configuration (reference) and the $\mathrm{iH}=0.0^{\circ}$ and $\mathrm{iH}=-2.0^{\circ}$ settings (necessary to carry out the interpolation). It is important to notice that the target lift value $C L=0.50$ is obtained with quite different angle of attack values, from $2.21^{\circ}$ for the tail-off configuration to $2.34^{\circ}$ for $\mathrm{iH}=0.0^{\circ}$ and $2.59^{\circ}$ for $\mathrm{iH}=-2.0^{\circ}$ configurations. Therefore, with different angles of attack, the flow around the wing and the empennage is somewhat different between the configurations and it may have a nonnegligible impact on the drag components.

Table 4. CRM WBH configuration - Downwash study - Lift and pitching moment coefficients.

\begin{tabular}{|c|c|c|c|}
\hline & $\boldsymbol{\alpha}$ & $\mathbf{C L}$ & $\mathbf{C M}$ \\
\hline Tail off & 2.21 & 0.5005 & -0.115 \\
\hline Tail iH $=\mathbf{0 . 0}$ & 2.34 & 0.4999 & -0.0471 \\
\hline Tail $\mathbf{i H}=\mathbf{- 2 . 0}^{\circ}$ & 2.59 & 0.5005 & +0.0824 \\
\hline Tail $\mathbf{i H}=\mathbf{- 0 . 7 2}^{\circ}$ & $\approx 2.43$ & $\approx 0.500$ & $\approx 0$ \\
\hline
\end{tabular}

Table 5. CRM WBH configuration - Downwash study - Near-field and far-field drag coefficients, evaluation of trim drag.

\begin{tabular}{|c|c|c|c|c|c|c|c|c|c|c|}
\hline & CL & CDp & CDf & CDnf & CDvp & CDv & CDi & CDw & CDff & CDsp \\
\hline Tail off & 0.5005 & 138.7 & 116.3 & 254.9 & 41.7 & 158 & 92.0 & 3.9 & 253.9 & 1.0 \\
\hline Tail iH $=\mathbf{0 . 0}$ & 0.4999 & 146.0 & 128.6 & 274.6 & 46.7 & 175.3 & 92.3 & 6.0 & 273.5 & 1.1 \\
\hline Tail $\mathbf{i H}=\mathbf{- 2 . 0}^{\circ}$ & 0.5005 & 164.9 & 127.4 & 292.3 & 52.2 & 179.7 & 98.2 & 13.5 & 291.4 & 1.1 \\
\hline Tail iH $=\mathbf{- 0 . 7 2}^{\circ}$ & $\approx 0.500$ & $\approx 152.7$ & $\approx 128.3$ & $\approx 281$ & $\approx 48.7$ & $\approx 176.9$ & $\approx 94.4$ & $\approx 8.7$ & $\approx 280$ & - \\
\hline Trim drag $\Delta\left(\mathbf{i H}=\mathbf{- 0 . 7 2}_{\mathbf{0}}^{\circ} \mathbf{0 f f}\right)$ & $\approx 0.0$ & $\approx 14.0$ & $\approx 12.0$ & $\approx 26.1$ & $\approx 7.0$ & $\approx 18.9$ & $\approx 2.4$ & $\approx 4.8$ & $\approx 26.1$ & - \\
\hline Trim drag sources \% & & & $46 \%$ & & $27 \%$ & & $9 \%$ & $18 \%$ & & \\
\hline
\end{tabular}

The total drag penalty for the trimmed configuration can be evaluated by interpolation at about 26 drag counts when compared to the tail-off configuration at $\mathrm{CL}=0.50$. The trim drag is composed of about 14 counts of pressure drag and about 12 counts of friction drag.

Even if generated through an approximate interpolation, it is very interesting to have access to the physical decomposition of the drag when estimating the drag penalty of the trimmed configuration. Over the 
26 drag counts of trim drag penalty at constant CL, about $46 \%$ come from the friction drag, $27 \%$ from the viscous pressure drag, $18 \%$ from the wave drag and $9 \%$ from the lift-induced drag. This very useful information is of prime importance for designers because it may largely help to reduce the trim drag of the aircraft.

\section{Mach and Reynolds numbers effects}

\section{A. Mach number effects}

The Mach sweep study corresponds to Test Case 2 of the DPW4. All the computations have been carried out with the medium grid on the $\mathrm{iH}=0^{\circ}$ tail configuration. This Mach sweep is computed for the following conditions:

- $\quad \mathrm{CL}=0.50$

- $\quad$ Mach number sweep: $\mathrm{Ma}=0.7,0.75,0.8,0.83,0.85,0.86,0.87$;

- $\quad$ Reynolds number $\mathrm{Re} / \mathrm{c}=5.10^{6}$.

The lift coefficient $\mathrm{CL}=0.50$ has been reached for all the calculations through a target lift algorithm available in the elsA software.

Table 6 and Table 7 give the lift, moment, near-field and far-field drag coefficients for the 7 Mach numbers which have been tested. The Mach number has been increased by increasing the infinite velocity magnitude.

Table 6. CRM WBH configuration - Mach number effect study - Lift and pitching moment coefficients.

\begin{tabular}{|c|c|c|c|c|c|}
\hline CRM tail $\left(\mathrm{iH}=0.0^{\circ}\right)$ & $\alpha$ & $\mathbf{C L}$ & CLwing & CLhtp & $\mathbf{C M}$ \\
\hline $\mathrm{Ma}=0.7$ & 3.01 & 0.4996 & 0.4451 & -0.0068 & -0.0608 \\
\hline $\mathrm{Ma}=0.75$ & 2.86 & 0.4998 & 0.4474 & -0.0087 & -0.0573 \\
\hline $\mathrm{Ma}=0.8$ & 2.67 & 0.5001 & 0.4507 & -0.0115 & -0.0515 \\
\hline $\mathrm{Ma}=\mathbf{0 . 8 3}$ & 2.49 & 0.5001 & 0.4534 & -0.0141 & -0.0465 \\
\hline $\mathrm{Ma}=0.85$ & 2.34 & 0.4999 & 0.4554 & -0.0161 & -0.0471 \\
\hline $\mathrm{Ma}=0.86$ & 2.25 & 0.4990 & 0.4564 & -0.018 & -0.0465 \\
\hline $\mathrm{Ma}=0.87$ & 2.25 & 0.4995 & 0.4575 & -0.0192 & -0.0458 \\
\hline
\end{tabular}

The pitching moment variation is about 0.015 , from -0.0458 at $\mathrm{Ma}=0.87$ to -0.0608 at $\mathrm{Ma}=0.7$. As all the computations have been performed at constant CL during the Mach sweep, the angle of attack variation (from $2.25^{\circ}$ at $\mathrm{Ma}=0.87$ to $3.01^{\circ}$ at $\mathrm{Ma}=0.7$ ) is quite noticeable, and this adds to the $\mathrm{CM}$ variation (Figure 19). 


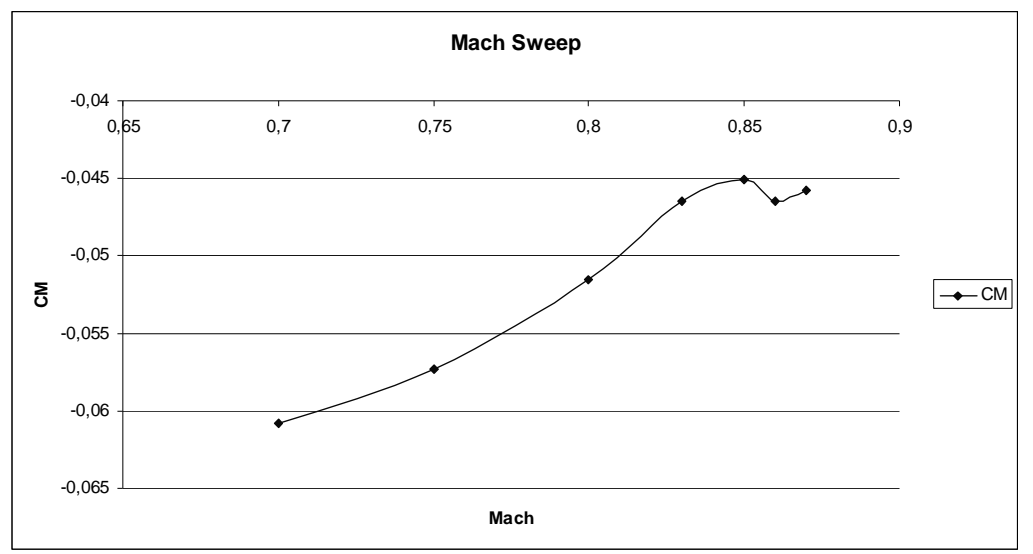

Figure 19. CRM wing / body / horizontal tail plane configuration - Mach sweep study - Pitching moment variation.

Table 7. CRM WBH configuration - Mach number effect study - Near-field and far-field drag coefficients.

\begin{tabular}{|c|c|c|c|c|c|c|c|c|c|c|}
\hline $\mathbf{C R M}_{\text {tail }}\left(\mathbf{i H}=\mathbf{0 . 0}^{\circ}\right.$ ) & $\mathbf{C L}$ & $\mathbf{C D p}$ & $\mathbf{C D f}$ & CDnf & CDvp & CDv & CDi & CDw & CDff & CDsp \\
\hline $\mathbf{M a}=\mathbf{0 . 7}$ & 0.4996 & 126.5 & 133.0 & 259.5 & 35.1 & 168.1 & 90.2 & 0.65 & 258.9 & 0.6 \\
\hline $\mathbf{M a}=\mathbf{0 . 7 5}$ & 0.4998 & 130.9 & 131.4 & 262.2 & 37.6 & 169.0 & 90.7 & 2.0 & 261.6 & 0.7 \\
\hline $\mathbf{M a}=\mathbf{0 . 8}$ & 0.5001 & 136.1 & 129.5 & 265.6 & 40.6 & 170.1 & 91.3 & 3.5 & 264.9 & 0.7 \\
\hline $\mathbf{M a}=\mathbf{0 . 8 3}$ & 0.5001 & 138.8 & 128.2 & 267.0 & 43.1 & 171.3 & 91.9 & 3.0 & 266.2 & 0.9 \\
\hline $\mathbf{M a}=\mathbf{0 . 8 5}$ & 0.4999 & 146.0 & 128.6 & 274.6 & 46.7 & 175.3 & 92.3 & 6.0 & 273.5 & 1.0 \\
\hline $\mathbf{M a}=\mathbf{0 . 8 6}$ & 0.4990 & 156.6 & 126.3 & 283.0 & 50.3 & 176.6 & 92.2 & 13.0 & 281.8 & 1.1 \\
\hline $\mathbf{M a}=\mathbf{0 . 8 7}$ & 0.4995 & 181.5 & 125.2 & 306.7 & 59.7 & 184.9 & 92.9 & 27.6 & 305.5 & 1.2 \\
\hline
\end{tabular}

The total drag variation due to the Mach increase is around 47 drag counts (see Figure 20). The friction drag coefficient decreases from 133 counts at $\mathrm{Ma}=0.7$ to 125.2 counts at $\mathrm{Ma}=0.87$ probably because of the appearance of flow separation. On the other hand, the pressure drag shows an increase of 55 counts. The far-field analysis permits to identify the pressure drag components which are responsible for such an increase. Obviously, the wave drag coefficient represents a great part of the pressure drag increase: about $49 \%$. Nevertheless, the viscous pressure drag rise is also important with a $\mathbf{4 5 \%}$ contribution (see Figure 21 ).

Figure 20 shows a particular characteristic at the point $\mathrm{Ma}=0.83$ where a light drop of the total drag is noticed while the general trend is an increase due to the Mach number rise. This phenomenon has also been observed by Boeing in this DPW4 Mach sweep context ${ }^{15}$, it seems to be particularly visible with the SST turbulence model. The far-field approach is very helpful in this case because it allows isolating the different physical components. Figure 21 represents some of the far-field drag coefficients, it clearly demonstrates that the light dimple at Ma $=0.83$ is 
entirely due to the $\mathrm{CDw}$ coefficient (shocks) while the viscous pressure drag (flow separations, wakes...) increases uniformly.

Figure 22 focuses on the span distribution of the wave drag for different Mach numbers from 0.75 to 0.85 . This far-field output permits to analyse the behaviour of the total drag between Mach $=0.8$ and Mach $=0.85$. First, it can be observed that the peak contribution to the wave drag moves towards the wing tip when the Mach number increases. When it is higher than 0.8 , the contributions of the outer part of the wing $(\mathrm{y}>20 \mathrm{~m})$ become significant. Then, the peak value and the area under the curve obtained for the Mach $=0.83$ computation are smaller than the same quantities at Mach $=0.8$. It justifies the existence of the dimple at Mach $=0.83$ when considering the wave drag curve and explains why the wave drag coefficient is weaker at this value than it is at Mach $=0.8$.

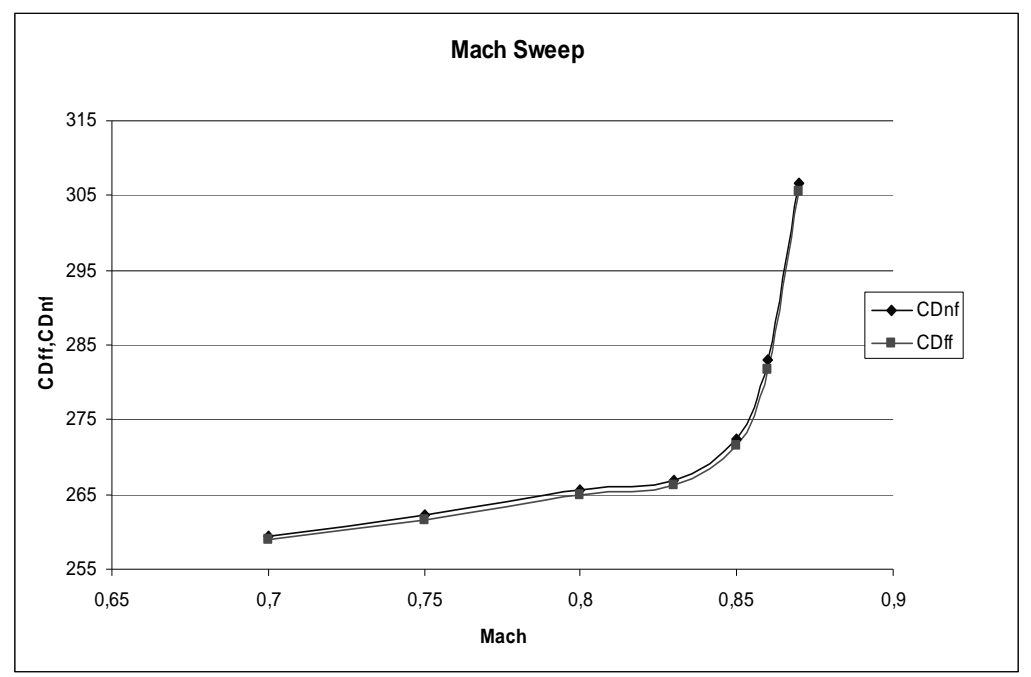

Figure 20. CRM wing / body / horizontal tail plane configuration - Mach sweep study - Total drag variation. 


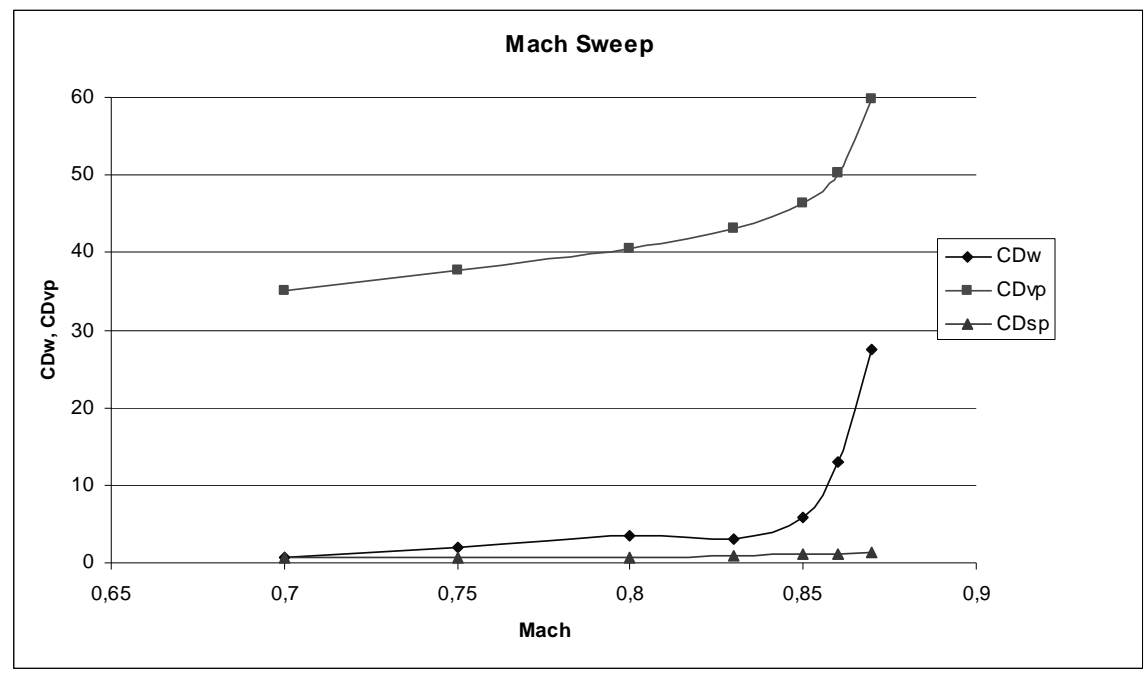

Figure 21. CRM wing / body / horizontal tail plane configuration - Mach sweep study - Far-field CDvp, CDw and CDsp coefficients.

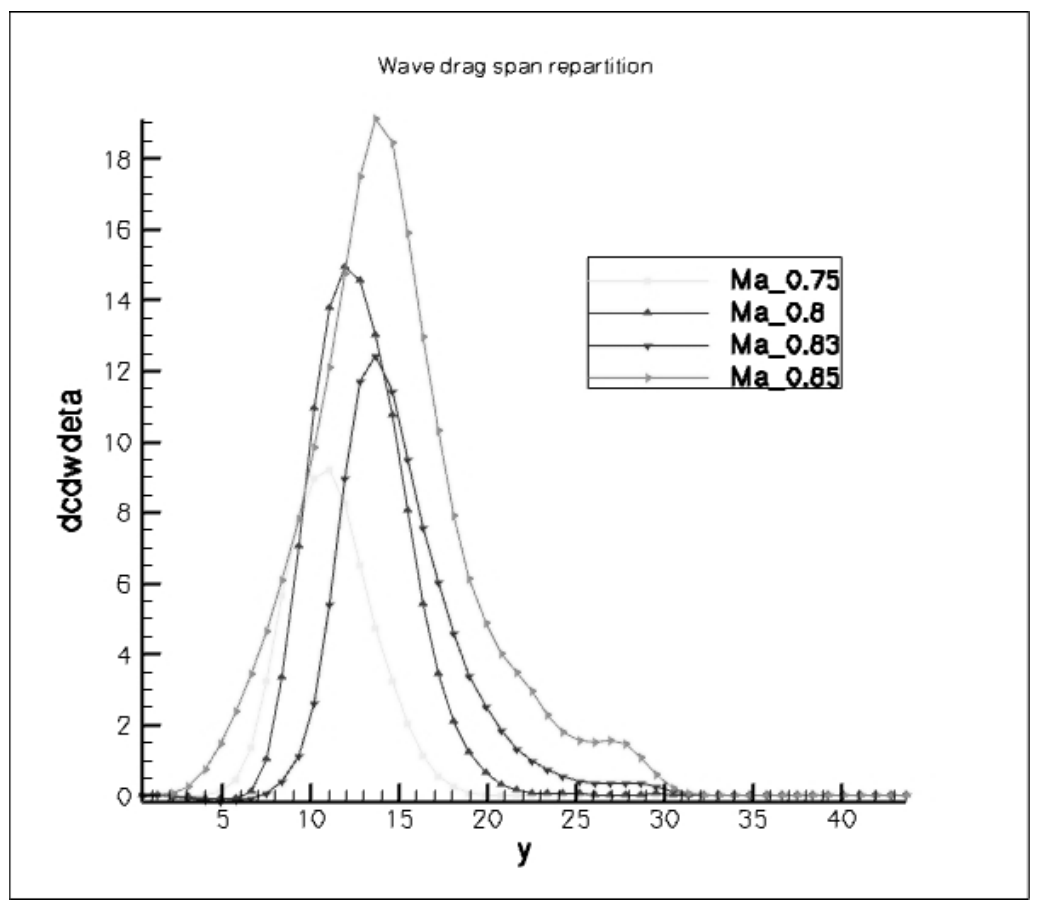

Figure 22. CRM wing / body / horizontal tail plane configuration - Mach sweep study - Span distribution of the wave drag contributions at different Mach numbers.

\section{B. Reynolds number effects}

The Reynolds number effect study corresponds to Test Case 3 of the DPW4. It uses the $\mathrm{iH}=0.0^{\circ}$ tail configuration. The study was carried out for the following conditions: 
- $\quad \mathrm{CL}=0.50$;

- $\mathrm{Ma}=0.85$;

- Reynolds number $\mathrm{Re} / \mathrm{c}=5.10^{6}$ corresponding to NASA Ames 11-ft transonic wind tunnel conditions and Reynolds number $=20.10^{6}$ corresponding to the NASA National Transonic Facility (NTF) at Langley.

In order to ensure a high convergence level, 1,800 iterations were done for all the computations as previously. The grid aimed at the $\mathrm{Re} / \mathrm{c}=5.10^{6}$ computation is the medium grid which has been used for the former grid convergence and downwash studies. For the Re/c $=20.10^{6}$ computation, Boeing provided another multiblock structured medium grid taking into account the higher Reynolds number and so the thinning down of the boundary layers. Therefore, even if both grids are very similar they are not exactly the same and it might introduce some numerical deviation in the aerodynamic comparison.

Table 8 gives the lift, moment and drag coefficients for the $\mathrm{Re} / \mathrm{c}=5.10^{6}$ and $\mathrm{Re} / \mathrm{c}=20.10^{6}$ calculations. Both computations have been performed at the same lift $\mathrm{CL}=0.5$. As a consequence, the angles of attack are different: $2.34^{\circ}$ for the first one and $2.13^{\circ}$ for the second one.

Table 8. CRM WBH configuration - Reynolds number effect study - Lift, pitching moment, near-field and far-field drag coefficients.

\begin{tabular}{|c|c|c|c|c|c|c|c|c|c|c|c|c|}
\hline CRM & $\boldsymbol{\alpha}$ & $\mathbf{C L}$ & $\mathbf{C M}$ & $\mathbf{C D p}$ & $\mathbf{C D f}$ & $\mathbf{C D n f}$ & $\mathbf{C D v p}$ & $\mathbf{C D v}$ & CDi & CDw & CDff & CDsp \\
\hline $\mathbf{R e}=5.10^{6}$ & 2.34 & 0.4999 & -0.0471 & 146.0 & 128.6 & 274.6 & 46.7 & 175.3 & 92.3 & 6.0 & 273.5 & 1.0 \\
\hline $\mathbf{R e}=20.10^{6}$ & 2.13 & 0.4990 & -0.0484 & 132.9 & 105.6 & 238.5 & 36.0 & 141.6 & 91.8 & 4.1 & 237.4 & 1.0 \\
\hline$\Delta$ & -0.213 & $\approx 0$ & -0.0013 & -13.1 & -23.0 & -36.1 & -10.7 & -33.7 & -0.5 & -1.9 & -36.1 & 0.0 \\
\hline
\end{tabular}

It can be noticed that the $\mathrm{CM}$ variation is about -0.0013 , from -0.0471 at $\operatorname{Re}=5.10^{6}$ to -0.0484 at $\operatorname{Re}=20.10^{6}$. The drag variation is about $36.1 \mathrm{drag}$ counts, the $\operatorname{Re}=20.10^{6}$ computation exhibiting the lowest value. This decrease of the drag value can be decomposed in a 13.1 count pressure drag drop (36\%) and a 23 count friction drag drop (64\%). The friction drag decrease is due to the skin friction coefficient drop generated by the increase of the Reynolds number. The far-field coefficients permit a finer analysis: the drop of pressure drag is made of $3.8 \%$ of lift-induced drag, $\mathbf{1 4 . 5 \%}$ of wave drag and $81.7 \%$ of viscous pressure drag contributions. The main effect on pressure drag is therefore caused by the thinning down of the boundary layers. The global drop of $36 \mathrm{drag}$ 
counts is essentially due to the viscous pressure drag and the friction drag components: these two coefficients represent $93.4 \%$ of the drag drop. As foreseen, the Reynolds number effects on drag coefficients are almost exclusively caused by viscous phenomena. The wave drag variation is more likely due to the difference between the angles of attack of both computations than to the Reynolds number increase.

\section{Conclusions}

This paper focuses on drag and pitching moment predictions for the $4^{\text {th }}$ AIAA DPW configuration using nearfield and far-field approaches. A special part is dedicated to the far-field methods at ONERA. The results presented in this article illustrate the current CFD capabilities to compute aerodynamic drag coefficients for transport aircraft. Uncertainties associated with numerical discretisation or grid quality have been assessed.

To complete this work, the multi-block structured grids provided by Boeing have been used and the DPW4 case 1.1 "Grid Convergence study", case 1.2 "Downwash study", case 2 "Mach Sweep" and case 3 "Reynolds Number Study" have been investigated with the ONERA-elsA solver in RANS mode using the Spalart-Allmaras turbulence model. Far-field drag analyses with the ONERA-ffd72 software have also been carried out.

The "Grid convergence study" exhibits an asymptotic evolution of pressure and skin friction drags with grid refinement, this effect is less evident for the pitching-moment component. Comparisons with Boeing RANS results on the same grids are satisfying as well for the drag components as for the pitching moment values.

The "Downwash study" is quite exhaustive and shows the capacity of the RANS approach to predict drag and pitching moment with high accuracy. These results permitted the trimmed drag value to be precisely estimated for an aircraft configuration at the appropriate lift coefficient and empennage angle. The far-field extraction gives the opportunity to identify the different sources that produce the trim drag (viscous pressure drag...).

The Mach and Reynolds numbers studies show very interesting variations of the drag coefficients. The far-field approach leads to a deeper insight by permitting one to evaluate the specific drag components which are mainly responsible for the total drag variation and to determine the contribution of each phenomenon.

In conclusion on the topic of drag prediction, good agreement has been observed between ONERA-elsA results and the near-field drag coefficients computed by other DPW4 participants such as Boeing, Airbus or DLR. Furthermore, the far-field drag approach gives the capability to quantify all the physical drag productions (viscous, 
wave and lift-induced contributions), to locate them and to estimate the deviation due to the numerical spurious drag. Therefore, the far-field post-processing clearly enables engineers to improve the aircraft design process.

\section{Acknowledgments}

The authors would like to thank D. Destarac who originated the far-field drag extraction software used in this study following a theory elaborated by J. van der Vooren.

\section{References}

${ }^{1}$ Esquieu, S., and Gazaix, M., "4 $4^{\text {th }}$ Drag Prediction Workshop: ONERA results”, http://aaac.larc.nasa.gov/tsab/cfdlarc/aiaadpw/Workshop4/presentations/DPW4 Presentations files/D1-8 DPWIV Onera jun20-21-2009.pdf, June 2009.

${ }^{2 .}$ Vassberg, J. C., DeHann, M. A., Rivers, S. M., and Wahls, R. A., "Development of a Common Research Model for Applied CFD Validation Studies", AIAA Paper, 2008-6919, 2008.

${ }^{3 .}$ Cambier, L., and Gazaix, M., "elsA: An Efficient Object-Oriented Solution to CFD Complexity", AIAA Paper, 2002-0108, Reno, 2002.

${ }^{4 .}$ Gazaix, M., Jolles, A., and Lazareff, M., "The elsA Object-Oriented Computational Tool for Industrial Applications”, ICAS Congress, 2002.

${ }^{5 .}$ Destarac, D., "Drag Extraction from Numerical Solutions to the Equations of Fluid Dynamics: the Far-field philosophy", presentation given at the 43eme Colloque d'Aérodynamique Appliquée de l'Association Aéronautique Astronautique de France, Poitiers, 10-12 March 2008.

${ }^{6}$ Van der Vooren, J., and Destarac, D., "Drag / Thrust Analysis of Jet-propelled Transonic Transport Aircraft; Definition of Physical Drag Components", Aerospace Science and Technology Vol.8, No7, October 2004.

${ }^{7}$ Van der Vooren, J., and Sloof, J. W., “CFD-based Drag Prediction: State-of-the-Art, Theory, Prospects", Lecture Notes, AIAA Professional studies, Course on Drag Prediction and Measurement, Portland, 1990, also NLR Technical Publication, TP 90247 L, 1990.

${ }^{8}$ Méheut, M., and Bailly, D., "Drag-Breakdown Methods from Wake Measurements", AIAA Journal, Vol. 46, No. 4, April 2008, pp. 847-862.

${ }^{9}$ Destarac, D., "Far-field Drag in Transonic Potential Flow: Analysis and Optimisation", Proceedings of the 1993 European Forum, Recent Developments and Applications in Aeronautical CFD, The Royal Aeronautical Society, Bristol, September 1$3,1993$. 
${ }^{10}$ Tognaccini, R., "Methods for Drag Decomposition, Thrust-Drag Bookkeeping from CFD Calculations", VKI Lecture Series 2003, CFD-based Aircraft Drag Prediction and Reduction, von Karman Institute for Fluid Dynamics, Rhode Saint Genèse, February 3-7, 2003.

${ }^{11 .}$ Jackson, A. P., and Martineau, D. G., "Computation of Wave and Viscous Drag Using Feature Extraction Techniques", AIAA paper, 2005-4626, June 2005.

12. Destarac, D., "Far-Field / Near-Field Drag Balance Applications of Drag Extraction in CFD", VKI Lecture Series 2003-02, CFD-Based Aircraft Drag Prediction and Reduction, National Institute of Aerospace, Hampton (VA), November 3-7, 2003.

${ }^{13}$ Schmitt, V., and Destarac, D., "Recent Progress in Drag Prediction and Reduction for Civil Transport Aircraft at ONERA", AIAA paper, 98-0137, January 1998.

${ }^{14}$ Esquieu, S., "Reliable Drag Extraction from Numerical Solutions: Elimination of Spurious Drag", AVT Symposium, RTOMP-AVT-147, Paper 42, Athens, Greece, 3-6 December 2007.

${ }^{15}$ Rider, B. J., and Tinoco, E. N., "CFL3D Analysis of the NASA Common Research Model for the 4th Drag Prediction Workshop",http://aaac.larc.nasa.gov/tsab/cfdlarc/aiaa-dpw/Workshop4/presentations/DPW4_Presentations_files/D16_DPW4Presentation_Solutions.pdf, June 2009.

${ }^{16 .}$ Vassberg, J. C., et al., "Summary of the Fourth AIAA CFD Drag Prediction Workshop", AIAA Paper, 2010-4547, Chicago, IL, June 2010. 